\title{
ESTUDIO FLORÍSTICO Y DE LA VEGETACIÓN DEL MUNICIPIO DE Buenavista de Cuéllar, Guerrero, México
}

\author{
Saddan Morales-Saldaña ${ }^{1,2}$, Emmanuel Martínez-Ambriz ${ }^{1}$ y Susana Valencia-Á. ${ }^{1}$ \\ ${ }^{1}$ Herbario de la Facultad de Ciencias, Universidad Nacional Autónoma de México, México, D.F., México \\ ${ }^{2}$ Autor para la correspondencia: saddanms@gmail.com
}

\begin{abstract}
Resumen: Estudios recientes han contribuido al conocimiento florístico del estado de Guerrero; sin embargo, aun existen regiones que no han sido estudiadas, como la parte norte de este, donde se ubica el municipio de Buenavista de Cuéllar, perteneciente a la región terrestre prioritaria 120 "Sierras de Taxco-Huautla"; por lo que se presenta el inventario florístico, un análisis de similitud florística y los tipos de vegetación de este muicipio. La lista florística incluye 611 especies, 331 géneros y 94 familias distribuidas en cinco tipos de vegetación: bosque de Juniperus, bosque de coníferas-Quercus, bosque de Quercus, bosque tropical caducifolio y pastizal inducido. El bosque tropical caducifolio es el más diverso con el $47.46 \%$ de las especies vegetales de Buenavista de Cuéllar. Magnoliophyta es el grupo más diverso con el 96.07\% de las especies encontradas. Las familias mejor representadas son Asteraceae (81 especies), Fabaceae (60), Apocynaceae (28) y Convolvulaceae (22). Se encontraron 29 especies endémicas para México, 11 para la Cuenca del Balsas y cuatro para Guerrero. El análisis de similitud florística de Buenavista de Cuéllar con la Sierra de Taxco, Sierra de Huautla y el Parque Nacional "General Juan N. Álvarez", muestra mayor similitud del sitio de estudio con la Sierra de Huautla.
\end{abstract}

Palabras clave: bosque de Quercus, bosque tropical caducifolio, región terrestre prioritaria 120, riqueza florística.

\begin{abstract}
Latest studies have contributed to the floristic knowledge of Guerrero state, however, there are still regions that have not been studied, for example, the north of Guerrero, where the municipality of Buenavista de Cuellar is located. This municipality belongs to the priority terrestrial region 120 "Sierras de Taxco-Huautla". Therefore, this paper presents the floristic inventory, an floristic similarity analysis and the vegetation types from the municipality of Buenavista del Cuellar. The floristic list includes 611 species, 331 genera, and 94 families distributed in five vegetation types: Juniper forest, pine-oak forest, oak forest, tropical deciduous forest and grassland. The tropical deciduous forest is the most diverse, with $47.62 \%$ of the vegetal species in Buenavista de Cuellar. Magnoliophyta is the group that provides the greatest specific richness with $96.07 \%$. The best represented families are Asteraceae (81 species), Fabaceae (60), Apocynaceae (28) and Convolvulaceae (22). Besides, there were found 29 endemic species for Mexico, 11 for Cuenca del Balsas and 4 for Guerrero. The floristic similarity analysis of Buenavista de Cuellar with the Sierra de Taxco, Sierra de Huautla and "General Juan N. Álvarez" National Park, showed the greatest similarity of the study site with Sierra de Huatla
\end{abstract}

Key words: floristic richness, priority terrestrial region 120, Quercus forest, tropical deciduous forest.

$\mathbf{E}^{1}$ 1 estado de Guerrero ocupa el quinto lugar en diversidad vegetal en el país con 5,529 especies, después de Oaxaca $(9,054)$, Chiapas $(7,830)$, Veracruz $(6,876)$ y Jalisco $(5,931)$ (García-Mendoza y Meave, 2011; Villaseñor y Ortíz, 2014); y es el tercer lugar en endemismos de plantas vasculares con 262 especies (Villaseñor y Ortíz, 2014). Sin embargo, el estado aún no cuenta con un inventario completo de su flora, lo que aunado a la rápida pérdida, degradación y fragmentación de los ecosistemas (de acuerdo con Sarukhán et al., 2009, el país conserva solo cerca del 50\% de su cobertura de vegetación original), demanda de un mayor esfuerzo en la exploración, recolecta, identificación y descripción de especies para lograr un inventario más completo de la biota estatal y nacional.

En el herbario de la Facultad de Ciencias de la UNAM (FCME) se desarrolla el proyecto de la Flora y Vegetación de la Cuenca del Balsas en su porción Guerrerense y, uno de los municipios que aun permanecía sin estudiar florísticamente era Buenavista de Cuéllar, colindante con el estado de Morelos e importante por pertenecer a la región terrestre prioritaria 120 "Sierras de Taxco-Huatla", que se caracteriza por su alta riqueza biológica en sus cañadas y una alta integridad ecológica (Arriaga et al., 2000).

Relacionados con el estudio florístico de Buenavista de 
Cuellar, está el de Catalán-Heverástico (1997) para la flora del Cañon de la Mano Negra, el de Martínez et al. (2004) para la porción guerrerense de la Sierra de Taxco y el de Dorado-Ramírez (2001) para la sierra de Huautla, los dos últimos enmarcados también en la región terrestre prioritaria 120. Por lo anterior, el presente trabajo tiene como objetivo presentar la lista florística, comparar la diversidad vegetal del municipio de Buenavista de Cuéllar con la de otras zonas de la misma región prioritaria y determinar los tipos de vegetación, para así contribuir al conocimiento de la flora y vegetación del estado de Guerrero.

\section{Materiales y métodos}

Área de estudio. El municipio de Buenavista de Cuéllar se localiza en la región norte del estado de Guerrero, entre los $18^{\circ} 22^{\prime}$ y $18^{\circ} 35^{\prime}$ de latitud norte y los $99^{\circ} 16^{\prime}$ y $99^{\circ} 32^{\prime}$ de longitud oeste (INEGI, 2009; Figura 1), con un área aproximada de $304 \mathrm{~km}^{2}$. Su altitud va de los 800 a los 2,200 m s.n.m. De acuerdo con Ferrusquía-Villafranca (2007) e INEGI (2009), el municipio pertenece a la provincia fisiográfica Sierra Madre del Sur y Rzedowski (1978) lo ubica en la provincia florística de la Depresión del Balsas. De acuerdo con INEGI (2009), predomina el clima cálido subhúmedo con lluvias en verano (Aw), con una temperatura mínima de $19^{\circ} \mathrm{C}$ y una máxima de $26^{\circ} \mathrm{C}$.

Trabajo de campo y de gabinete. Se realizaron diez visitas a la zona de estudio, de agosto de 2011 a septiembre de 2012, con intervalos aproximados de un mes, y con una duración de al menos tres días cada una, durante las cuales se realizaron recorridos a través de los senderos del municipio. Con apoyo del programa Google Earth, así como del sistema de información geográfica ArcGis 9 (ESRI, 2010) y de las cartas

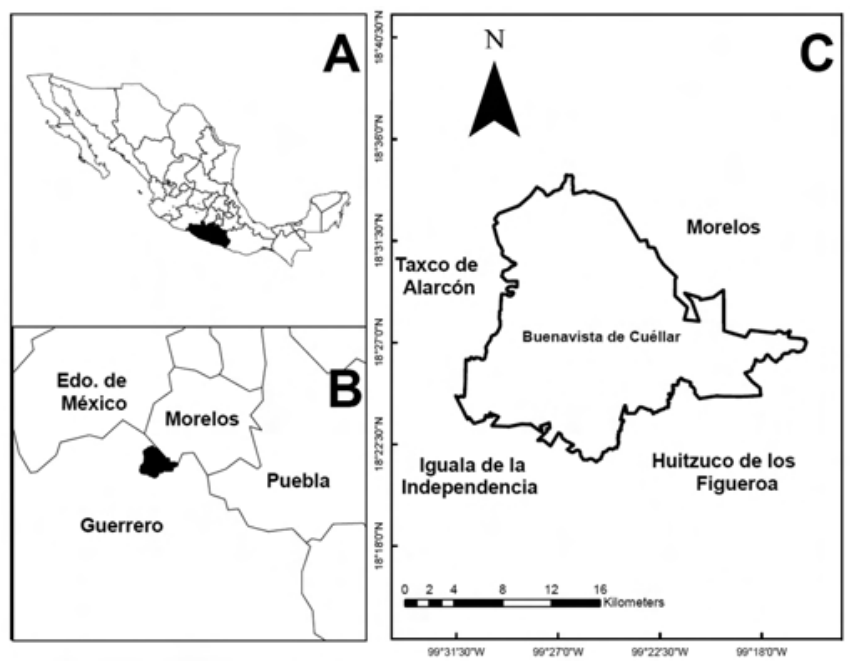

Figura 1. A) Ubicación del estado de Guerrero en México, B) Localización del municipio de Buenavista de Cuéllar dentro del estado de Guerrero y C) Municipio de Buenavista de Cuéllar. topográficas E14A68, E14A69 y E14A78 escala 1:50,000, se corroboró que en todos los tipos de vegetación del área de estudio se hubieran hecho recolectas durante todas las visitas. Los ejemplares se recolectaron y procesaron de acuerdo con lo señalado por Lot y Chiang (1986) y fueron depositados en el Herbario de la Facultad de Ciencias de la Universidad Nacional Autónoma de México (FCME). La identificación de las especies se realizó utilizando monografías, descripciones originales y floras: Flora de Guatemala (Standley y Steyermark, 1946,1949,1952; Standley y Williams, 1961,1966,1975; Gentry y Standley, 1974; Nash y Williams, 1976), Flora Fanerogámica del Valle de México (Calderón de Rzedowski y Rzedowski, 2001), Flora Mesoamericana (Davidse et al., 2009). Flora de Veracruz (Gómez-Pompa, 1978-1991; Sosa, 1992-2005; Castillo-Campos, 2005), Flora del Bajío y regiones adyacentes (Rzedowski y Calderón de Rzedowski, 1993-2013). Flora Novo-Galiciana (Anderson W.R., 1983, 1984, 1987, 1989, 1992, 2001), Flora del Valle de Tehuacán-Cuicatlán (Dávila et al., 1993-2000; Kelly et al., 2001-2004; Novelo, 2004-2007, Medina-Lemos, 20072012) y Flora de Guerrero (Diego-Pérez y Fonseca, 19972013). Asimismo, se tuvo el apoyo de los especialistas de algunas familias para la identificación del material.

Adicionalmente, se revisó el material de herbario previamente recolectado en el municipio de Buenavista de Cuéllar y depositado en el Herbario Nacional (MEXU) y en el herbario de la Facultad de Ciencias de la UNAM (FCME). También se consultaron las bases de datos incluidas en la Red Mundial de Información sobre Biodiversidad (REMIB), disponibles en la página electrónica de la Comisión Nacional para el Conocimiento y Uso de la Biodiversidad (CONABIO). Esto con el objetivo de localizar recolectas hechas, con anterioridad, en el municipio por otros colectores.

Los datos obtenidos de las recolectas realizadas y consultadas se integraron en una base de datos en Microsoft Access, con la finalidad de organizar la información. También se determinó el estado de conservación de las especies usando el criterio de la lista roja de la Unión Internacional para la Conservación de la Naturaleza (IUCN, 2012) y la Norma Oficial Mexicana NOM-059-SEMARNAT-2010 (SEMARNAT, 2010).

Con el objeto de comparar la flora de la zona de estudio con la citada en estudios anteriores en la región terrestre prioritaria 120, se realizó un análisis de similitud que incluyó la lista florística de la Sierra de Huautla (Dorado-Ramírez, 2001), la de la Sierra de Taxco (Martínez et al., 2004), la del Parque Nacional "Juan N. Álvarez" (Bustamante-García, 2012) y la obtenida en este trabajo, con las que se elaboró una matriz de presencia-ausencia de especies, donde el 1 representa presencia y 0 ausencia. La matriz se analizó utilizando el sistema UPGMA, utilizando el programa NTSYSpc 2.11T (Rohlf, 2004) mediante el índice de similitud de Jaccard. Los resultados se presentan por medio de un dendrograma. Las especies cultivadas y las sinonimias de las floras previamente reportadas fueron omitidas. 
La ubicación taxonómica de las especies en sus respectivas familias siguió los criterios de Mickel y Smith (2004) para Lycophyta y Monilophyta; McVaugh (1992) para Gimnospermas y APG III (APG, 2009) para Magnoliophyta. La lista florística se organizó en Lycophyta, Monilophyta, Gimnospermas y Magnoliophyta, y dentro de esta última en Monocotiledóneas y Eudicotiledóneas. En todos los grupos se siguió un orden alfabético en un sistema jerarquizado de familia, género y especie.

Con base en la información generada por el Centro Canadiense de Teledetección et al. (2010), se elaboró un mapa de vegetación del área de estudio en el sistema de información geográfica ArcGis 9 (ESRI, 2010), que posteriormente fue corroborado en campo.

\section{Resultados}

Riqueza florística. Se recolectaron 1,163 números, que aunados con los registros de herbarios de material previamente recolectado en el área, resultaron en 94 familias, 331 géneros y 611 especies para el municipio de Buenavista de Cuéllar (Apéndice 1). El grupo que mayor aporte tiene a la riqueza florística es Magnoliophyta con 587 especies (96.07\%), de las cuales 80 son monocotiledóneas (13.1\%) y 507 eudicotiledóneas (82.97\%). Las Lycophyta y Moni-

Cuadro 1. Riqueza de grupos taxonómicos de la Flora del municipio de Buenavista de Cuéllar, Guerrero.

\begin{tabular}{lccc}
\hline & Familias & Géneros & Especies \\
\hline $\begin{array}{l}\text { Lycophyta y } \\
\text { Monilophyta }\end{array}$ & $4(4.25 \%)$ & $9(2.71 \%)$ & $22(3.60 \%)$ \\
Gimnospermas & $2(2.12 \%)$ & $2(0.60 \%)$ & $2(0.32 \%)$ \\
Eudicotiledóneas & $73(77.65 \%)$ & $276(83.38 \%)$ & $507(82.97 \%)$ \\
Monocotiledóneas & $15(15.95 \%)$ & $44(13.3 \%)$ & $80(13.09 \%)$ \\
Total & $\mathbf{9 4}$ & $\mathbf{3 3 1}$ & $\mathbf{6 1 1}$ \\
\hline
\end{tabular}

lophyta representan el $3.6 \%$ de las especies, el $2.71 \%$ de los géneros y el $4.25 \%$ de las familias citadas. Mientras que las Gimnospermas representan únicamente el $0.32 \%$ de las especies en el municipio (Cuadro 1).

Las familias y géneros con mayor riqueza de especies se presentan en el cuadro 2. En este se observa que el $53.1 \%$ de la flora está representada por 14 familias: Asteraceae (81), Fabaceae (60), Apocynaceae (28), Convolvulaceae (22), Solanaceae (17), Euphorbiaceae (16), Malvaceae (15), Lamiaceae (15), Cyperaceae (14), Boraginaceae (13), Rubiaceae (13), Bromeliaceae (12), Malpighiaceae (11) y Bignoniaceae (8); el otro $46.81 \%$ se distribuye en las 80 familias restantes. El $20 \%$ de las especies de Buenavista de Cuéllar están contenidas en el $4.2 \%$ de los géneros presentes en el área de estudio. El género más diverso es Ipomoea con 18 especies, seguido de Cuphea con 12 especies, el tercer lugar lo ocupan Stevia y Bursera con diez especies.

El $60.45 \%$ de las especies de Buenavista de Cuéllar son hierbas, los árboles están representados por el $17 \%$ de las mismas, los arbustos constituyen el $15.84 \%$ y el $6.7 \%$ son bejucos. La familia con más especies en el estrato arbóreo es Fabaceae (14), seguida por Burseraceae (10), Fagaceae y Malvaceae (7). Mientras que las familias más ricas, tanto en el estrato arbustivo como en el herbáceo, son Asteraceae, con 21 especies de arbustos y 58 especies de hierbas y, Fabaceae con 16 y 24 respectivamente. La familia Apocynaceae es la más rica en bejucos, con 14 especies.

Con base en el trabajo de Rodríguez-Jiménez et al. (2005) se registraron cuatro especies endémicas de Guerrero, 11 de la Cuenca del Balsas y 29 especies endémicas de México (Apéndice 1). Se encontraron cinco especies bajo alguna categoría de riesgo con base en la Norma Oficial Mexicana NOM-059-SEMARNAT-2010 para la Protección de Especies Nativas de México (SEMARNAT, 2010; Cuadro 3), y ocho especies se encuentran en la Lista Roja de especies en

Cuadro 2. Familias y géneros mejor representados en la flora del área de estudio

\begin{tabular}{lccc}
\hline Familia & Géneros (especies) & Género & Número de especies \\
\hline Asteraceae & $46(81)$ & Ipomoea & 18 \\
Fabaceae & $37(60)$ & Cuphea & 12 \\
Apocynaceae & $15(28)$ & Stevia & 10 \\
Colvolvulaceae & $5(22)$ & Bursera & 10 \\
Solanaceae & $6(17)$ & Salvia & 9 \\
Euphorbiaceae & $7(16)$ & Solanum & 9 \\
Malvaceae & $9(15)$ & Cyperus & 8 \\
Lamiaceae & $4(15)$ & Melampodium & 7 \\
Cyperaceae & $6(14)$ & Oxalis & 7 \\
Boraginaceae & $9(13)$ & Tillandsia & 7 \\
Rubiaceae & $8(13)$ & Begonia & 7 \\
Malpighiaceae & $9(11)$ & Euphorbia & 6 \\
Bromeliaceae & $3(12)$ & Polygala & 6 \\
Bignoniaceae & $8(8)$ & Total & 6 \\
Total & $\mathbf{1 7 2 ( 3 2 5 )}$ & & $\mathbf{1 2 2}$ \\
\hline
\end{tabular}


peligro de la Unión Internacional para la conservación de la Naturaleza (IUCN, 2012; Cuadro 4).

Se encontró un nuevo registro de la familia Caricaceae para la flora de Guererro, Jarilla nana, especie sólo conocida con anterioridad en los estados de Hidalgo, Guanajuato, Jalisco, México, Michoacán, Nayarit, Zacatecas y el Distrito federal (Bentham, 1848; Díaz y Lomelí, 1992; Calderón de Rzedowski y Lomelí, 1993; McVaugh, 2001; García-Mendoza y Meave, 2011; Carvahlo y Renner, 2012).

Como resultado del análisis de similitud, se encontraron 138 especies exclusivas de Buenavista de Cuéllar, 156 compartidas entre la Sierra de Taxco, Buenavista de Cuéllar y la Sierra de Huautla, y solamente 25 se encuentran en las cuatro áreas analizadas (Acacia pennatula, Arbutus xalapensis, Asclepias glaucescens, Bessera elegans, Bouvardia chrysantha, Buddleja sessiliflora, Castilleja tenuiflora, Commelina tuberosa, Crotalaria pumila, Ipomoea purpurea, Lantana urticifolia, Lopezia racemosa, Loeselia glandulosa, Ludwigia octovalvis, Mimosa albida, Phytolacca icosandra, Piqueria trinervia, Solanum laurifolium, Tagetes lucida, Tridax coronopifolia, T. mexicana, Valeriana urticifolia, Viguiera cordata, Vitis tiliifolia y Wigandia urens).

La comparación de la riqueza específica del municipio con los trabajos florísticos analizados, muestra que la Sierra de Huautla comparte el $26.06 \%$ de sus especies con Buenavista de Cuéllar, mientras que la Sierra de Taxco el 25.72\% (cuadro 5). El coeficiente de similitud más alto (0.23) se presenta entre la Sierra de Huautla y Buenavista de Cuéllar, lo cual quiere decir que son florísticamente más similares en relación con demás áreas (Figura 2).

Vegetación. Se registran el bosque de Quercus, bosque tropical caducifolio, bosque de Juniperus, bosque de coníferas-Quercus y pastizal inducido (Figura 3). Además existen zonas agrícolas y urbanas con una extensión aproximada de $56 \mathrm{~km}^{2}$. El bosque tropical caducifolio es el más diverso con 291 especies, seguido del bosque de Quercus con 254. Los tipos de vegetación con menor riqueza son el bosque de $J u$ -

Cuadro 3. Especies que se encuentran bajo alguna categoría de riesgo en la NOM-ECOL-059-2010.

\begin{tabular}{|c|c|c|}
\hline Especie & Familia & Estatus \\
\hline $\begin{array}{l}\text { Bouvardia loeseneriana } \\
\text { Standl. }\end{array}$ & Rubiaceae & $\begin{array}{c}\operatorname{Pr} \text { (sujetas a } \\
\text { protección especial) }\end{array}$ \\
\hline $\begin{array}{l}\text { Coryphantha elephantidens } \\
\text { (Lem.) Lem. }\end{array}$ & Cactaceae & A (amenazada) \\
\hline $\begin{array}{c}\text { Crusea hispida (Mill.) } \\
\text { B.L. Rob. }\end{array}$ & Rubiaceae & $\begin{array}{c}\operatorname{Pr}(\text { sujetas a } \\
\text { protección especial) }\end{array}$ \\
\hline $\begin{array}{c}\text { Dalbergia congestiflora } \\
\text { Pittier }\end{array}$ & Fabaceae & $\begin{array}{l}\mathrm{P} \text { (en peligro de } \\
\text { extinción) }\end{array}$ \\
\hline $\begin{array}{l}\text { Sideroxylon capiri } \\
\text { (A. DC.) Pittier }\end{array}$ & Sapotaceae & A (amenazada) \\
\hline
\end{tabular}

Cuadro 4. Especies que se encuentran en la lista roja con base en IUCN.

\begin{tabular}{ccc}
\hline Especie & Familia & Estatus IUCN \\
\hline Juniperus flaccida & Cupressaceae & Preocupación menor \\
Arbutus xalapensis & Ericaceae & Preocupación menor \\
Coryphantha elephantidens & Cactaceae & Preocupación menor \\
Crotalaria pumila & Fabaceae & Preocupación menor \\
Lonchocarpus caudatus & Fabaceae & Preocupación menor \\
Mimosa albida var. albida & Fabaceae & Preocupación menor \\
Swietenia humilis & Meliaceae & Vulnerable \\
Tillandsia recurvata & Bromeliaceae & Preocupación menor
\end{tabular}

niperus con 141 especies, el bosque de coníferas-Quercus con 79 y el pastizal con 14 especies (Cuadro 6).

Bosque de coníferas-Quercus.- Cuenta con una extensión de $13.48 \mathrm{~km}^{2}$, equivalente al $4.42 \%$ del territorio municipal. Se desarrolla por arriba de los 1,800 m s.n.m., en las zonas montañosas del este y noreste del área de estudio. Se pueden encontrar tres estratos. El arbóreo se caracteriza por presentar elementos de 5-25 m, con especies como Alnus jorullensis, Arbutus xalapensis, Quercus candicans, $Q$. castanea, Q. magnoliifolia, Q. urbanii. Pinus pringlei y Xylosma intermedia. El estrato arbustivo está poco desarrollado, su altura es de 1-2.5 m, destacan Calliandra houstoniana, Dodonaea viscosa, Fuchsia microphylla, Karwinskia mollis, Salvia sessei y Solanum erianthum. En el estrato herbáceo dominan especies de $5 \mathrm{~cm}$ hasta $1 \mathrm{~m}$ de alto, como Achimenes woodii, Aldama dentata, Allium glandulosum, Asclepias auriculata, Euphorbia ariensis, Pinaropappus roseus y Valeriana urticifolia. La única epífita presente es Polypodium cryptocarpon, mientras que Ipomoea dimorpophylla es el único bejuco registrado.

Bosque de Quercus.- Tiene una extensión de 107 km², equivalente al 35.14\% del área de estudio. Se desarrolla en las zonas montañosas del municipio, desde los 1,400 hasta los 2,200 m s.n.m., aunque más comúnmente entre los 1,500 y $1,800 \mathrm{~m}$. Presenta tres estratos, el arbóreo, con una altura

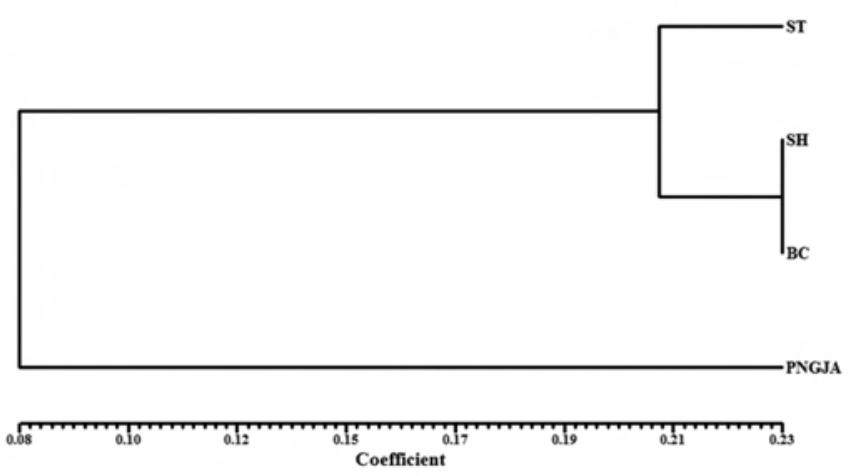

Figura 2. Dendrograma resultante del análisis de agrupamiento (UPGMA) de similitudes florísticas entre Sierra de Taxco (ST), Sierra de Huautla (SH), Buenavista de Cuéllar (BC) y P.N. "General Juan N. Álvarez" (PNGJNA). Coeficiente de correlación cofenética $\mathrm{r}=0.94$. 


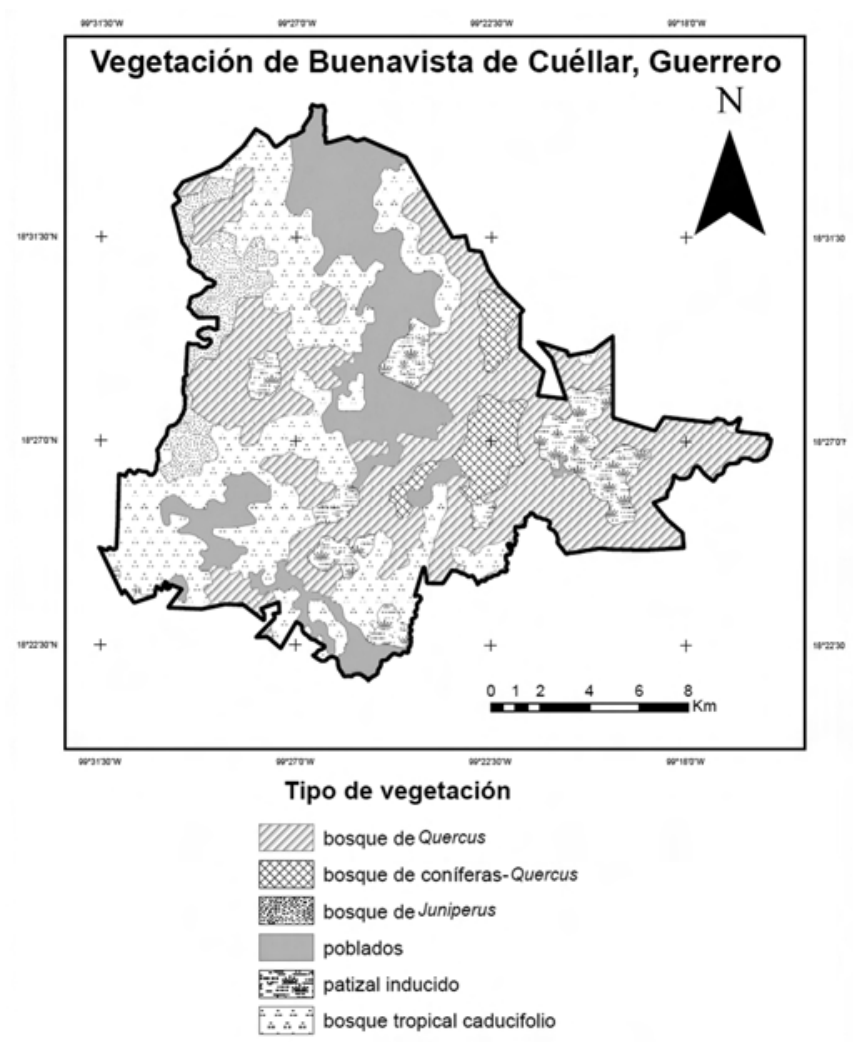

Figura 3. Mapa de vegetación de la zona de estudio. Modificado de Centro Canadiense de Teledetección et al. (2010).

entre 10 y 15 m, está formado por especies como Arbutus xalapensis, Arctostaphylos oppositifolia y Quercus magnoliifolia, este último es el elemento dominante fisonómico de esta comunidad. Entre los arbustos destacan Brongniartia montalvoana, Chromolaena odorata, Mimosa albida, Montanoa bipinnatifida, Salvia sessei y Solanum americanum; mientras que en el estrato herbáceo dominan Adiantum andicola, Bessera elegans, Bletia gracilis, Evolvulus alsi-

Cuadro 5. Comparación de la riqueza florística del municipio de Buenavista de Cuéllar con otros estudios florísticos.

\begin{tabular}{ccccc}
\hline Estudio & $\begin{array}{c}\text { Área } \\
\left(\mathbf{k m}^{2}\right)\end{array}$ & $\begin{array}{c}\text { No. de } \\
\text { especies }\end{array}$ & $\begin{array}{c}\text { No. de } \\
\mathbf{s p p} / \mathbf{k m}^{2}\end{array}$ & $\begin{array}{c}\text { Especies } \\
\text { compartidas } \\
\text { en relación con } \\
\text { Buenavista de } \\
\text { Cuéllar }\end{array}$ \\
\hline $\begin{array}{c}\text { Sierra de Taxco } \\
\text { (Martínez et al., 2004) }\end{array}$ & 730 & 1,384 & 1.895 & $\begin{array}{c}356 \\
(25.72 \%)\end{array}$ \\
$\begin{array}{c}\text { Sierra de Huautla } \\
\text { (Dorado-Ramírez, 2001) }\end{array}$ & 591 & 936 & 1.58 & $\begin{array}{c}244 \\
(26.06 \%)\end{array}$ \\
$\begin{array}{c}\text { P.N. "General Juan } \\
\text { N. Álvarez" }\end{array}$ & 528 & 395 & 0.75 & $\begin{array}{c}80 \\
(20.25 \%)\end{array}$ \\
$\begin{array}{c}\text { (Bustamante-García, 2012) } \\
\text { Buenavista de Cuéllar } \\
\text { (en este estudio) }\end{array}$ & 304 & 611 & 2 & 611 \\
\hline
\end{tabular}

Cuadro 6. Riqueza florística por tipo de vegetación en el municipio de Buenavista de Cuéllar. El número entre paréntesis indica los taxa exclusivos para cada tipo de vegetación

\begin{tabular}{lccc}
\hline Tipo de vegetación & Familias & Géneros & Especies \\
\hline Bosque de Quercus & $64(7)$ & $164(65)$ & $253(214)$ \\
Bosque de pino-encino & $38(5)$ & $60(18)$ & $79(37)$ \\
Bosque de Juniperus & $49(5)$ & $89(23)$ & $142(65)$ \\
Bosque tropical caducifolio & $65(16)$ & $190(101)$ & $290(185)$ \\
Pastizal inducido & $7(0)$ & $13(0)$ & $13(0)$ \\
\hline
\end{tabular}

noides, Ranunculus sierrae-orientalis y Rhodosciadium diffusum. Entre las epífitas están Tillandsia achyrostachys, T. caput-medusae, T. hintoniana, T. ionantha y T. recurvata. Bosque tropical caducifolio.- Abarca un área de $86.63 \mathrm{~km}^{2}$, el $28.42 \%$ del territorio municipal. Se encuentra desde los 1,000 hasta los 1,700 m s.n.m., más comúnmente entre los 1,200 y $1,600 \mathrm{~m}$. Se desarrolla en las partes bajas del municipio, sobre laderas y lomeríos con suelos poco profundos. El estrato arbóreo incluye especies que oscilan entre los 3 y 10(-15) m de altura, como Actinocheita filicina, Bunchosia palmeri, Bursera ariensis y Haematoxylum brasiletto, aunque en ocasiones se pueden encontrar árboles emergentes que llegan a alcanzar hasta los $15 \mathrm{~m}$, como Ipomoea arborescens, Ceiba aesculifolia y Sideroxylon capiri. El estrato arbustivo varía de un sitio a otro, ya que se encuentra en función del dosel arbóreo, aunque generalmente se encuentra muy desarrollado con alturas de $50 \mathrm{~cm}$ hasta $3 \mathrm{~m}$, con especies como Buddleja sessiliflora, Croton adspersus y Pittocaulon bombycophole. El estrato herbáceo está bien desarrollado en temporada de lluvias por especies con alturas de $10 \mathrm{~cm}$ hasta $1.5 \mathrm{~m}$, como Acalypha phleoides, Achimenes grandiflora, Begonia monophylla, Dorstenia drakena y Ruellia hookeriana. El número de bejucos es muy numeroso y entre las especies más comunes se encuentran Arrabidaea patellifera, Blepharodon mucronatum, Callaeum coactum, Cissus cacuminis y Heteropterys brachiata. Se registró a Tillandsia achyrostachys, T. ionantha y T. recurvata como epífitas. Entre las hemiparásitas se encuentran Phoradendron pedicellatum, Psittacanthus calyculatus y P. schideanus.

Bosque de Juniperus.- Se localiza al noroeste y oeste del municipio, colinda con Taxco de Alárcon. Abarca solamente $5.66 \%$ del territorio municipal con una extensión de $17.215 \mathrm{~km}^{2}$. Se desarrolla desde los 1,500 hasta los $2,000 \mathrm{~m}$ s.n.m., aunque más comúnmente entre los 1,800 y 2,000 m. Se localiza principalmente en laderas, lomeríos, así como en algunos terrenos planos aunque muy expuestos. El estrato arbóreo presenta especies de 3-8 $\mathrm{m}$ de altura, con algunos individuos emergentes hasta de $15 \mathrm{~m}$, como Juniperus flaccida, Bursera glabrifolia, Ceiba aesculifolia, Erythrina lanata, Eysenhardtia platycarpa y Ficus petiolaris. En temporadas de lluvias, el estrato arbustivo está bien desarrollado y se observan individuos de 1-2.5 $\mathrm{m}$ de altura como 
Bouvardia loeseneriana, Cnidoscolus rostratus, Euphorbia schlechtendalii y Galphimia glauca; mientras que el estrato herbáceo se encuentra bien representado en temporada de lluvias y consta de elementos desde los $5 \mathrm{~cm}$ hasta los $2 \mathrm{~m}$ de altura, como Astrolepis leavis, Cuphea avigera, Echeveria gibbiflora, Elytraria imbricata y Stevia aschenborniana. Además de las especies mencionadas, en las laderas más húmedas también se encuentran especies como Arisaema dracontium, Bdallophytum americanum, Peperomia cavispicata y Sedum quevae. Los principales bejucos son Clematis dioica, Nissolia fruticosa, Pithecoctenium crucigerum y Serjania racemosa. Se pueden encontrar epífitas como Tillandsia achyrostachys, T. ionantha y T. schiedeana.

Pastizal inducido.- Se localiza en la parte sur, este y centro del municipio. Ocupa una extensión de $24.44 \mathrm{~km}^{2}$, lo que equivale al $8.03 \%$ del territorio municipal. Se desarrolla entre $\operatorname{los} 1,200$ y 1800 m s.n.m., en terrenos con poca pendiente. Esta comunidad es inducida en el municipio con la finalidad de realizar actividades ganaderas; presenta un estrato herbáceo poco diverso, algunas de las especies son Digitaria sp., Evolvulus alsinoides, Ixophorus sp., Macroptilium gibbosifolium, Paspalum notatum y Tagetes filifolia; también hay algunos elementos arbóreos esparcidamente distribuidos para proporcionar sombra, como Acacia pennatula y Quercus glaucoides; los arbustos que se presentan pertenecen a Dodonaea viscosa, Mimosa albida, Waltheria americana y Wigandia urens.

\section{Discusión}

Para este trabajo se registraron 94 familias, 331 géneros y 611 especies. Si se consideran los trabajos de Fernández et al. (1998) y Villaseñor y Ortiz (2014), el número de especies encontradas en Buenavista de Cuéllar representa el 13.73\% de todas las especies de la Cuenca del Balsas, el $11.03 \%$ de las especies de Guerrero y el $2.79 \%$ de las especies de México. Con base en estos resultados, es importante señalar que el $11.03 \%$ de la diversidad vegetal reportada para Guerrero está representada en el $0.47 \%$ de su territorio.

Para la flora de Buenavista de Cuéllar destaca Magnoliophyta, que concentra el $96.05 \%$ de la riqueza de esepcies vegetales. Las eudicotiledóneas son el grupo que se encuentra mejor representado con $83.27 \%$, dentro del cual, 14 familias concentran el $53.19 \%$ de las especies de este estudio y cinco de ellas (Asteraceae, Fabaceae, Euphorbiaceae, Lamiaceae y Solanaceae) se incluyen entre las 11 familias mejor representadas para México (Villaseñor, 2003). Estos patrones en la riqueza de las familias no difieren con otros estudios florísticos de regiones de Guerrero (Calónico-Soto, 2001; Jiménez et al., 2003; Martínez et al., 2004; Valencia-Ávalos et al. 2011).

En este trabajo se registraron diez especies del género Bursera, equivalente al $21.27 \%$ de las 47 especies reportadas para la Cuenca del Balsas (Fernández et al., 1998; Guevara-Féfer, 2010) y $11.36 \%$ de las 88 especies para
México (Rzedowski et al., 2005; Rzedowski y Calderón de Rzedowski, 2006; Guevara-Féfer, 2010; León de la Luz y Pérez-Navarro, 2010; Medina-Lemos, 2013). Otra cifra que es interesante resaltar, es que el $26.47 \%$ de las especies del género Solanum reportadas para Guerrero (34 especies con base en Fuentes-Peryañez, 2009), están presentes en el municipio de Buenavista de Cuéllar; asimismo, el género Cyperus está representado con el 15\% de sus especies en el área de estudio, respecto a las 51 citadas para Guerrero por Diego-Pérez (1997).

Con base en el trabajo de Villaseñor y Espinoza-García (2004), en el estado de Guerrero se encuentran 126 especies introducidas y en este trabajo se registran seis de ellas (Annona cherimola, Centella erecta, Cyperus esculentus, Melia azederach, Nicotiana glauca y Sonchus oleraceus), equivalente al $4.76 \%$ de dicha flora. Asimismo, $0.9 \%$ de la flora del área de estudio está representada por especies introducidas.

La comparación de la flora del municipio de Buenavista de Cuéllar con la reportada para la Sierra de Huautla y para la Sierra de Taxco, muestra que se comparten 244 y 356 especies respectivamente, y que 138 se citan por primera vez para la región prioritaria 120 .

La similitud florística de la Sierra de Huautla, Buenavista de Cuéllar y Sierra de Taxco resulta congruente con la pertenencia de estas tres regiones a la provincia florística de la Depresión del Balsas, mientras que la poca similitud (0.08) con la zona del Parque Nacional "General Juan N. Álvarez" se debe a que se localiza en la provincia florística de las serranías meridionales.

El bosque de Quercus es la comunidad con mayor extensión en el municipio, aunque no es el más diverso, debido a que su riqueza de 253 especies es superada ligeramente por la del bosque tropical caducifolio con 290, el cual ocupa el segundo lugar en extensión. La mayor extensión del bosque de Quercus no es sorprendente, ya que se trata de un bosque dominado por $Q$. magnoliifolia, una especie caducifolia adaptada a condiciones estacionales tropicales, como es el caso de la Cuenca del Balsas.

El pastizal inducido del municipio se distribuye de forma fragmentada en las zonas aledañas a los poblados, este es una comunidad vegetal secundaria, producto principalmente del desmonte del bosque tropical caducifolio para las actividades ganaderas que se realizan en el municipio, las cuales han causado cambios en la composición y estructura de la comunidad original. La baja diversidad florística encontrada para esta comunidad, particularmente de gramíneas, se puede explicar por la preferencia de ciertas especies para alimentar al ganado y por el sobrepastoreo.

\section{Conclusiones}

En el municipio Buenavista de Cuéllar se encontraron 611 especies de plantas vasculares, 138 de las cuales no se habían reportado para la región terrestre prioritaria 120, lo que auna- 
do a las listas florísticas de Dorado-Ramírez (2001) y Martínez et al. (2004) dan un total de 2,117 especies de plantas vasculares para dicha región. Así se resalta la importancia de este estudio debido a que complementa el conocimiento florístico para esta zona, lo cual es reforzado con el bajo índice de similitud florística entre las áreas estudiadas en la región.

La topografía del municipio favorece la presencia de cuatro tipos de vegetación naturales, donde el bosque de Quercus es el más extenso. Tanto el bosque de Quercus, como el bosque de coníferas y Quercus son las comunidades mejor conservadas por los pobladores de Buenavista de Cuéllar, quienes les confieren alto valor por proporcionarles beneficios ambientales, por lo que estos bosques no padecen tala o alteraciones considerables.

En el municipio se registraron cuatro especies endémicas para Guerrero, 11 para la Cuenca del Balsas y 29 para México, así como el hallazgo por primera vez de Jarilla nana para el estado de Guerrero y 12 especies con algún grado de protección de acuerdo con SEMARNAT (2010) e IUCN (2012), lo que sugiere, se trata de una región que aunque presenta actividades ganaderas y de recreación, posee áreas dignas de ser protegidas para su conservación.

\section{Agradecimientos}

Al personal del Herbario Nacional (MEXU) por otorgar las facilidades para la revisión de la colección. A los especialistas Oscar Hinojosa Espinoza (Asteraceae), Martha Martínez Gordillo (Euphorbiaceae y Lamiaceae), Ramiro Cruz Durán (Fabaceae), Rosa María Fonseca Juárez (Pinaceae), Ana Belem Adame González (Selaginellaceae), Eduardo Pérez García (Orchidaceae) y Lucio Lozada (Asclepiadaceae). A José Luis Villaseñor por la revisión preliminar de la lista florística y a Jaime Jiménez por la lectura y sugerencias para mejorar este escrito. A Ramón Cuevas y a un revisor anónimo por las sugerencias y crítica constructiva para mejorar este escrito.

\section{Literatura citada}

Anderson W.R. Ed. 1983, 1984, 1987, 1989, 1992, 2001. Flora Novo-Galiciana. University of Michigan Press, University of Michigan Herbarium, Ann Arbor.

APG. The Angiosperm Phylogeny Group. 2009. An update of the Angiosperm Phylogeny Group classification for the orders and families of flowering plants: APG III. Botanical Journal of the Linnean Society 161:105-121.

Arriaga L., Espinoza J.M., Aguilar C., Martínez E., Gómez L. y Loa E. (Coords.). 2000. Regiones Terrestres Prioritarias de México. Comisión Nacional para el Conocimiento y uso de la Biodiversidad, México, D.F.

Bentham G. 1848. Carica nana sp. nov. Plantae Hartwegianae. G. Pamplin. Londres.

Bustamante-García R. 2012. Estudio florístico en el Parque Nacional "General Juan N. Álvarez" Guerrero, México.Tesis deMaestría, Facultad de Ciencias, Universidad Nacional Autónoma de México, México, D.F. 156 pp.
Calderón de Rzedowski G. y Lomelí S. J.J. 1993. Caricaceae. Flora Del Bajío y Regiones Adyacentes 17:1-12.

Calderón de Rzedowski G. y Rzedowski J. 2001. Flora fenerogámica del Valle de México. Instituto de Ecología, A.C., Comisión Nacional para el Conocimiento y Uso de la Biodiversidad, Pátzcuaro.

Calónico-Soto J. 2001. Contribución a la flora de la Cuenca del Río Balsas en su parte oriental, Tecoyo y sus alrededores: municipio de Alpoyeca, Guerrero. Tesis licenciatura, Facultad de Ciencias, Universidad Nacional Autónoma de México, México, D.F. 69 pp.

Carvalho F. A. y Renner S. S. 2012. A dated phylogeny of the papaya family (Caricaeae) reveals the crop's closest relatives and the family's biogeographic history. Molecular Phylogenetics and Evolution 65:46-53.

Castillo-Campos G. Ed. 2005. Flora de Veracruz. Instituto de Ecología, A.C., Centro de Investigaciones Tropicales de la Universidad Veracruzana, Xalapa, Veracruz, México.

Catalán-Heverástico C. 1997. Flora del Cañón de la Mano Negra, Municipios de Buenavista de Cuéllar e Iguala, Guerrero, México. Tesis de Maestría, Colegio de Posgraduados, Montecillo. 66 p.

Centro Canadiense de Teledetección, Sector Ciencias de la Tierra, ministerio de Recursos Naturales, Comisión Nacional para el Conocimiento y Uso de la Biodiversidad, Comisión Nacional Forestal, Instituto Nacional de Estadística y Geografía, Servicio Geológico de Estados Unidos. 2010. Cobertura del suelo de México, 2005, a 250 metros. Edición: 1.0. Centro Canadiense de Teledetección, Servicio Geológico de Estados Unidos, Instituto Nacional de Estadística y Geografía Comisión para la Cooperación Ambiental. <http://www.conabio.gob.mx/infor $\mathrm{macion} / \mathrm{metadata} / \mathrm{gis} / \mathrm{nalcmsmx} 05 \mathrm{gw} . \mathrm{xml}$ ?_httpcache=yes\&_ $\mathrm{xsl}=/ \mathrm{db} / \mathrm{metadata} / \mathrm{xsl} / \mathrm{fgdc} \_$html.xsl\&_indent=no> (consultado 18 de febrero de 2013)

Davidse G., Sousa S., M. Knapp S. y Chiang F. 2009. Cucurbitaceae a Polemoniaceae. Flora mesoamericana 4 (parte 1):1-855.

Dávila A.P.D., Villaseñor R.J.L., Medina L.R. y Téllez V.O. Eds.1993-2000. Flora del Valle de Tehuacán-Cuicatlán. Instituto de Biología, Universidad Nacional Autónoma de México, México, D.F.

Díaz L.C. L. y Lomelí S.J.A. 1992. Revisión del género Jarilla Rusby (Caricaceae). Acta Botanica Mexicana 20:77-99.

Diego-Pérez N. 1997. Cyperaceae. Flora de Guerrero 5:1-170.

Diego-Pérez N. y Fonseca R.M. 1997-20013. Flora de Guerrero. Facultad de Ciencias Universidad Nacional Autónoma de México, México, D.F.

Dorado-Ramírez O. R. 2001. Sierra de Huautla-Cerro Frío, Morelos: Proyecto de reserva de la biosfera. Universidad Autónoma del Estado de Morelos, Centro de Investigación en Biodiversidad y Conservación. Informe final SNIB-CONABIO proyecto No. Q025. México D. F.

ESRI. 2010. Maps throughout this book were created using ArcGIS $®$ software by Esri. ESRI, Redlands.

Fernández N. R., Rodríguez C. J., Arreguín S. M. L. y Rodríguez J. A. 1998. Listado florístico de la Cuenca del Río Balsas, México. Polibotánica 9:1-151.

Ferrusquía-Villafranca I. 2007. Ensayo sobre su caracterización y significación biológica. En: Luna V.I., Morrone J.J. y Espinosa D. Eds. Biodiversidad de la Faja Volcánica Transmexicana, pp. 7-24, Comisión Nacional para el Conocimiento y Uso de la Biodiversidad, Universidad Nacional Autónoma de México, México D.F. 
Fuentes-Peryañez C. 2009. La familia Solanaceae en los municipios Atenango del Río y Copalillo, Guerrero. Tesis de Licenciatura, Facultad de Ciencias. Universidad Nacional Autónoma de México. México, D.F. 147 pp.

García-Mendoza A. J. y Meave J. A. Eds. 2011. Diversidad Florística de Oaxaca: De Musgos a Angiospermas (Colecciones y Listas de Especies). Universidad Nacional Autónoma de México-Comisión Nacional para el Conocimiento y Uso de la Biodiversidad. México, D.F.

Gentry J. L. y Standley P.C. 1974. Flora of Guatemala. Parte X, números 1 y 2. Fieldiana: Botany 24:1-151.

Gómez-Pompa A.1978-1991. Flora de Veracruz. Instituto de Ecología, A.C., Xalapa.

Guevara-Féfer. 2010. Una nueva especies de Bursera (Burseraceae), endémica de la cuenca baja del río Balsas en los estados de Michoacán y Guerrero, México. Acta Botanica Mexicana 92:119-128.

INEGI. Instituto Nacional de Estadística y Geografía. 2009. Buenavista de Cuéllar, Guerrero. Prontuario de información geográfica de los Estados Unidos Mexicanos. Clave geoestadística 12015. Instituto Nacional de Estadística y Geografía, Aguascalientes.

IUCN. International Union for Conservation of Nature. 2012. Red List of Threatened Species. V. 2012.2. <http://www.iucnredlist. org> (consultado 22 de febrero de 2013).

Jiménez R.J., Martínez G.M., Valencia A.S., Cruz D.R., Contreras J.J.L., Moreno G.E. y Calónico S.J. 2003. Estudio florístico del municipio Eduardo Neri, Guerrero. Anales del Instituto de Biología, Universidad Nacional Autónoma de México, Serie Botánica 74:79-142.

Kelly L.M., Ochoterena H. y Medina L.R. Eds. 2001-2004. Flora del Valle de Tehuacán-Cuicatlán. Instituto de Biología, Universidad Nacional Autónoma de México, México, D.F.

León de la Luz J.L. y Pérez-Navarro J. J. 2010. Dos nuevos taxa de Bursera (Burseraceae) de Baja California Sur, México. Acta Botanica Mexicana 91:37-49.

Lot A. y Chiang F. 1986. Manual de Herbario. Consejo Nacional de la Flora de México, A.C. México, D.F.

Martínez G. M., Cruz D. R., Castrejón R.J F., Valencia A. S., Jiménez R. J. y Ruiz-Jiménez C.A. 2004. Flora Vascular de la porción guerrerense de la Sierra de Taxco, Guerrero, México. Anales del Instituto de Biología, Universidad Nacional Autónoma de México, Serie Botánica 75:105-189.

McVaugh R.1992. Gymnosperms and Pteridophytes. Flora NovoGaliciana 17:1-467.

McVaugh R. 2001. Ochnaceae to Loasaceae. Flora Novo-Galiciana 3:1-751

Medina L.R. 2007-2012. Flora del Valle de Tehuacán-Cuicatlán. Instituto de Biología Universidad Nacional Autónoma de México, D.F.

Medina-Lemos R. 2013. Una nueva species de Bursera (Burseraceae) del sur de México. Acta Botanica Mexicana 103:9-25.

Mickel J. T. y Smith A. R. 2004. The Pteridophytes of México. Memoirs of the New York Botanical Garden 88:1-1092.

Nash D.I. y Williams L.O. 1976. Flora of Guatemala. Parte XII. Fieldiana: Botany 24:1-603.

Recibido: 3 de diciembre de 2013

Aceptado: 29 de marzo de 2014
Novelo R.A. 2004-2007. Flora del Valle de Tehuacán-Cuicatlán. Instituto de Biología Universidad Nacional Autónoma de México, México, D.F.

Rodríguez-Jiménez C., Fernández-Nava R., Arreguín-Sánchez M. L., Rodríguez-Jiménez A.2005. Plantas vasculares endémicas de la Cuenca del río Balsas, México. Polibotánica 20:73-99.

Rohlf J. 2004. NTSYS-pc 2.11T. Numerical Taxonomy and Multivariate Analysis System.2.11T Exeter Software. Applied Bioestatistics, Nueva York.

Rzedowski J. 1978. Vegetación de México. Limusa, México, D.F.

Rzedowski J., Medina-Lemos R. y Calderón de Rzedowski G. 2005. Inventario de conocimiento taxonómico, así como de la diversidad y el endemismo regionales de las especies mexicanas de Bursera (Burseraceae). Acta Botanica Mexicana 70:85-111.

Rzedowski J. y Calderón de Rzedowski G. 2006. Dos especies nuevas de Bursera (Burseraceae) de México. Acta Botanica Mexicana 74:169-178.

Rzedowski J y Calderón de Rzedowski G. 1993-2013. Flora del Bajio y de Regiones Adyacentes. Instituto de Ecología, A.C. Pátzcuaro.

Sarukhán J., Koleff P., Carabias J., Soberón J., Dirzo R., Llorente-Bousquets J., Halffter G., González R., March I., Mohar A., Anta S. y de la Maza J. 2009. Capital Natural de México. Síntesis: conocimiento actual, evaluación y perspectivas de sustentabilidad. Comisión Nacional para el Conocimiento y Uso de la Biodiversidad, México, D.F.

SEMARNAT. Secretaría de Medio Ambiente y Recursos Naturales. 2010. Norma Oficial Mexicana NOM-059-SEMARNAT2010. Protección ambiental-Especies nativas de México deflora y fauna silvestres-Categorías de riesgo y especificaciones para su inclusión, exclusión o cambio-Listade especies en riesgo.

Sosa V. 1992-2005. Flora de Veracruz. Instituto de Ecología, A.C., Xalapa.

Standley P. C. y Steyermark J.A. 1946. Flora of Guatemala. Parte IV. Fieldiana: Botany 24:1-493.

Standley P. C. y Steyermark J.A. 1949. Flora of Guatemala. Parte VI. Fieldiana: Botany 24:1-440

Standley P. C. y Steyermark J.A. 1952. Flora of Guatemala. Parte III. Fieldiana: Botany 24:1-432.

Standley P. C. y Williams L.O. 1961. Flora of Guatemala. Parte VII. Fieldiana: Botany 24:1-281.

Standley P. C. y Williams L.O. 1966. Flora of Guatemala. Parte VIII, números 1 y 2. Fieldiana: Botany 24:1-210.

Standley P. C. y Williams L.O. 1975. Flora of Guatemala. Parte XI, números 1 a 3. Fieldiana: Botany 24:1-151.

Valencia-Ávalos S., Cruz-Durán R., Martínez-Gordillo M y Jiménez-Ramírez. J. 2011. La flora del municipio Atenango del Río, estado de Guerrero, México. Polibotánica 32:9-39.

Villaseñor J. L. 2003. Diversidad y distribución de las Magnoliophyta de México. Interciencia 28:160-167

Villaseñor J.L y Espinoza-García F. J. 2004. The alien flowering plants of Mexico. Diversity and Distributions 10:113-123.

Villaseñor J.L y Ortíz E. 2014. Biodiversidad de las plantas con flores (División Magnoliophyta) en México. Revista Mexicana de Biodiversidad 85(supl.):134-142 
FLORA Y VEGETACión de BuenAVISTA DE CuÉLlar

Anexo 1. Lista florística del municipio de Buenavista de Cuéllar, Guerrero.

La lista florística se presenta en orden alfabético por familia, género y especie y esta dividida en los siguientes grupos: Lycophyta, Monilophyta, Gimnospermas y Magnoliophyta, dividida a su vez en Eudicotiledoneas y Monocotiledoneas. + = endémica de Méxi$\mathrm{Co}^{*}{ }^{*}=$ endémica de Guerrero, ${ }^{\circ}=$ no había sido reportada para la región terrestre prioritaria 120 . bQ = bosque de Quercus, bcQ = bosque de coníferas-Quercus, btc $=$ bosque tropical caducifolio y bJ = bosque de Juniperus. $\bullet=$ Registros de herbario $($ MEXU y FCME) VU = vulnerable, $\mathrm{LC}=$ preocupación menor, categorías establecidas con base en la IUCN (2012). A = Amenazada, $\mathrm{P}=$ En peligro de extinción y $\operatorname{Pr}=$ Sujetas a protección especial, Categorías establecidas con base en NOM-059-SEMARNAT-2010. C = especies presentes tanto en la Sierra de Taxco, Sierra de Huautla y Buenavista de Cuéllar.

\section{Lycophyta}

Selaginellaceae

1. Selaginella pallescens (C.Presl) Spring, A. Adame G. s.n. (FCME), hierba, bQ.

2. S. ribae Valdespino, A. Adame G. s.n. (FCME), hierba, bJ.

3. S. rupincola Underw., A. Adame G.s.n. (FCME), hierba, bJ, bcQ.

4. S. wrightii Hieron, A. Adame G.s.n. (FCME), hierba, bQ.

\section{Monilophyta}

\section{Anemiaceae}

5. CAnemia hirsuta (L.) Sw., S. Morales S. 156 (FCME), hierba, bQ.

6. A. karwinskyana (C.Presl) Prantl, S. Morales S. 372 (FCME), hierba, bQ.

7. A. mexicana Klotzsch, S. Morales S. 1194 (FCME), hierba, btc.

\section{Polypodiaceae}

8. ${ }^{\circ}$ Polypodium cryptocarpon Fée, S. Morales S. 1021 (FCME), hierba, bcQ.

9. P. furfuraceum Schltdl. \& Cham., S. Morales S. 1152 (FCME), hierba, bcQ.

\section{Pteridaceae}

10. Adiantum andicola Liebm., S. Morales S. 379 (FCME), hierba, bQ.

11. (C) A. concinnum Humb et Bonpl. ex Willd., S. Morales S. 554 (FCME), hierba, bQ.

12. A. patens Willd., S. Morales S. 339 (FCME), hierba, bQ.

13. ${ }^{\circ}$ A. shepherdii Hook., S. Morales S. 163 (FCME), hierba, bQ.

14. Astrolepis laevis (M.Martens \&Galeotti) Mickel, S. Morales S. 986 (FCME), hierba, bJ.

15. A.sinuata (Lag. ex Sw.) D.M.Benham \& Windham, S. Morales S. 977 (FCME), hierba, bJ.

16. Bommeria pedata (Sw.) E.Fourn., S. Morales S. 345 (FCME), hierba, bQ.

17. ${ }^{\circ}$ Cheilanthes decomposita (M.Martens \& Galeotti) Fée, S. Morales S. 704 (FCME) hierba, bQ.

18. C. farinosa (Forssk.) Kaulf., S. Morales S. 569 (FCME), hierba, bQ.

19. ${ }^{\circ}$ C. longipila Baker, S. Morales S. 745 (FCME), hierba, bQ.

20. Notholaena candida (M. Martens et Galeotti) Hook. S. Morales S. 675 (FCME) hierba, bJ.

21. N. galeotti Fée, S. Morales S. 6 (FCME), hierba. bQ.

22. Pellaea ovata (Desv.)Weath., S. Morales S. 1167 (FCME), hierba, bcQ.

\section{Gimnospermas}

\section{Cupressaceae}

23. Juniperus flaccida Slchtdl., S. Valencia A.3426 (FCME), árbol, bJ, LC.

\section{Pinaceae}

24. Pinus pringlei Shaw, S. Morales S. 825 (FCME), árbol, bcQ.

\section{Magnoliophyta \\ Eudicotiledóneas \\ Acanthaceae}

25. Aphelandra sp., S. Morales S. 1198 (FCME), hierba, bQ.

26. CCarlowrightia arizonica A.Gray, S. Morales S. 766 (FCME), arbusto, btc, b)

27. (C). neesiana (Schauer ex Nees) T.F.Daniel, S. Morales S. 756 (FCME), sufrútice, btc.

28. C. pectinata Brandegee, S. Morales S. s.n. (FCME), hierba, bQ.

29. Dyschoriste hirsutissima (Nees) Kuntze, S. Morales S. 798 (FCME), sufrútice, btc, bJ

30. CElytraria imbricata (Vahl.) Pers., S. Morales S. 913 (FCME), hierba, bJ.

31. Justicia fulvicoma Schltdl. \& Cham., O. Tabares s.n. (FCME), sufrútice, bQ. 
Anexo 1. Continuación

32. Ruellia fruticosa Sessé \& Moc., S. Morales S. 970 (FCME), arbusto, bJ

33. R. hookeriana (Nees) Hemsl., S. Morales S. 87, 897 (FCME), hierba, btc .

34. • R. nudiflora (Engelm. \& A.Gray) Urb., J.C. Soto 8732 (MEXU), hierba, btc.

35. Stenandrium dulce (Cav.) Nees, S. Morales S. 817 (FCME), hierba, bQ, bJ.

36. CTretramerium nervosum Nees, S. Morales S. 850 (FCME), hierba, bQ.

\title{
Amaranthaceae
}

37. (Comphrena serrata L., S. Morales S. 214, 709, 601 (FCME), hierba, btc, bQ, bJ.

38. Iresine diffusa Bonpl. ex Willd., A. Andrade R. 3 (FCME), hierba, bQ.

\section{Anacardiaceae}

39. (C)+ Actinocheita filicina (DC.) F.A.Barkley, S. Valencia A. 5229 (FCME), S. Morales S. 78, 245, 968 (FCME), árbol, btc, bJ. 40. CSpondias purpurea L., V. Acosta A. S.n., S. Valencia A. 4865 (FCME), S. Morales S.843 (FCME), árbol, btc.

\section{Annonaceae}

41. (CAnnona cherimola Mill., S. Valencia A. 4868 (FCME), S. Morales S. 257, 1039 (FCME), árbol, btc, bJ, bQ. 42. A. reticulata L., S. Morales S. 332, 1093 (FCME), árbol, btc.

\author{
Apiaceae \\ 43. Centella erecta (L.f.) Fernald, S. Morales S. 997 (FCME), hierba, bQ. \\ 44. • Donnellsmithia mexicana (B.L. Rob) Mathias et Constance, F. Terán y S. Vázquez 246 (FCME), hierba, bQ. \\ 45. - Eryngium ghiesbreghtii Decne., R. M. Fonseca 831 (FCME), hierba, btc. \\ 46. ${ }^{\circ}$ E. globosum Hemsl., S. Morales S. 799 (FCME), hierba, bJ. \\ 47. ${ }^{\circ}$ E. spiculosum Hemsl., F. Lorea 3208 (FCME), hierba, bQ. \\ 48. Micropleura renifolia Lag., S. Valencia A. 4835 (FCME), hierba, bQ. \\ 49. $+{ }^{\circ}$ Neogoezia breedlovei Constance, S. Morales S. 555 (FCME), hierba, bQ. \\ 50. • Prionosciadium. diversifolium Rose, F. Terán 421 (FCME), hierba, btc. \\ 51. P. nelsonii J.M. Coult. \& Rose, S. Morales S. 67, 940, 985 (FCME), hierba, btc, bQ, bJ. \\ 52. ${ }^{\circ}$ P. thapsoides (DC.) Mathias, S. Valencia A. 4824 (FCME), hierba, bQ. \\ 53. ${ }^{\circ}$ Rhodosciadium diffusum (J.M.Coult. \& Rose) Mathias \& Constance, S. Morales S. 908 (FCME), hierba, bJ.
}

\section{Apocynaceae}

54. Asclepias auriculata Kunth, S. Morales S. 197, 146 (FCME), hierba, bcQ, bQ.

55. (CA. curassavica L., G. Castillo 7 (FCME), S. Morales S. 811 (FCME), hierba, bQ, btc.

56. A. glaucescens Kunth, S. Valencia A. 4837 (FCME), S. Morales S 882 (FCME), hierba, bQ, bcQ.

57. • A. Iynchiana Fishbein, E. Molseed 467 (MEXU), hierba, btc.

58. A. ovata M.Martens \& Galeotti, S. Morales S. 1044 (FCME), hierba, bcQ.

59. CBlepharodon mucronatum (Schltdl.) Decne, V. Acosta 6 (FCME), S. Morales S. 818 (FCME), bejuco, bQ, btc.

60. (C) Cascabela ovata (Cav.) Lipold, S. Morales S. 219, 876, 916 (FCME), árbol, btc.

61. C. peruviana (Pers.) Raf., S. Morles S. s.n. (FCME), arbusto, btc.

62. (C) . thevetioides (Kunth) Lippold, S. Valencia A. 3401, 4829 (FCME), S. Morales S. 73 (FCME), árbol, bQ.

63. (C) Cynanchum foetidum (Cav.) Kunth, S. Morales S. 30 (FCME), bejuco, btc.

64. ${ }^{\circ}$ C. ligulatum (Benth.) Woodson, S. Morales S. 237 (FCME), bejuco, btc.

65. Dictyanthus pavonii Decne., S. Morales S. 338, 73 (FCME) bejuco, bQ, btc.

66. + Funastrum pannosum Schltr., S. Morales S. 989 (FCME), bejuco, btc.

67. F. sp., S. Valencia A. 5265 (FCME), bejuco, btc.

68. Gonolobus sp., S. Morales S. s.n (FCME), bejuco.,btc.

69. CHaplophyton cimicidum A.DC. S. Morales S. 21, 32 (FCME), hierba, btc.

70. Laubertia contorta (M.Martens \& Galeotti) Woodson. S. Morales S. 1181 (FCME), bejuco, btc.

71. $\bullet$ Mandevilla holosericea (Sessé \& Moc.) J.K.Williams, J. C. Soto y S. Aureoles 8926 (MEXU), bejuco, btc.

72. ${ }^{\circ}$ M. karwinskii (Müll.Arg.) Hemsl., M. Luján 1 (FCME), arbusto, bQ.

73. • M. syrinx Wood., J.C. Soto 8926 (MEXU), bejuco, btc.

74. Marsdenia zimapanica Hemsl, S. Valencia A. 4825 (FCME), bejuco, bQ.

75. Matelea crenata (Vall.) Woodson, S. Valencia A. 5230 (FCME), bejuco, btc.

76. Matelea chrysantha (Greenm.) Woodson, S. Morales S. 153, 1046 (FCME), bejuco, bQ, btc.

77. Polystemma guatemalense (Schltr.)W.D.Stevens, S. Morales S. 18 (FCME), bejuco, btc.

78. CPlumeria rubra L., S. Morales S. s.n. (FCME), árbol, bJ. 
Flora y VEGETACIÓN DE BuENAVista de CuÉllaR

Anexo 1. Continuación

79. ${ }^{\circ}$ Tabernaemontana donnell-smithi Rose, S. Morales S. s.n (FCME), árbol, btc.

80. CT. odontadeniiflora A.O.Simoes \& M.E.Endress, S. Morales S. s.n. (FCME), árbol, btc.

81. - T. tomentosa (Greenm) A.O.Simões \& M.E.Endress, C. Catalán y F. Terán 660 (FCME), arbusto, btc.

\section{Aristolochiaceae}

82. Aristolochia orbicularis Duch, S. Morales S. 1099 (FCME), hierba, bJ, btc.

\section{Asteraceae}

83. Acmella repens (Walter) R.K.Jansen, S. Casas V. s.n. (FCME), hierba, btc.

84. ${ }^{\circ}$ A. radicans (Jacq.) R.K.Jansen, S. Morales S. 476 (FCME), hierba, btc.

85. (C)Ageratum corymbosum Zuccagni, S. Morales S. 355 (FCME), hierba, btc.

86. Ageratina choricephala (B.L.Rob) R.M.King \& H.Rob., S. Morales S., 720 (FCME), hierba, bQ.

87. A. sp., S. Morales S. 585, 616 (FCME), hierba, bQ, bJ.

88. (CAldama dentata La Llave, S. Morales S., 783 (FCME), S. Valencia A. 5269 (FCME), hierba, btc, bcQ.

89. Alloispermum integrifolium (DC.) H.Rob., Y. Dávila 3 (FCME), arbusto, bQ.

90. Baccharis salicifolia (Ruiz \& Pav.) Pers., S. Morales S. 116 (FCME), arbusto, bQ.

91. Barkleyanthus salicifolius (Kunth) H.Rob et Brettell, J. Gotes 4 (FCME), arbusto, bcQ.

92. CBidens odorata Cav., S. Morales S. 775 (FCME), hierba, bcQ.

93. (C) Calea ternifolia Kunth, S. Morales S. 387 (FCME), arbusto, bQ.

94. ${ }^{\circ}$ C. urticifolia (Mill.) DC., S. Morales S. 596 (FCME), arbusto, bQ.

95. Conyza canadensis (L.) Cronquist, S. Valencia A. 4880 (FCME), hierba, btc.

96. ${ }^{\circ}$ Critoniopsis uniflora (Sch.Bip.) H.Rob., S. Morales S. 790 (FCME), arbusto, bQ.

97. ${ }^{\circ}$ Chromolaena collina (DC.) R.M.King \& H.Rob, S. Morales S. 617 (FCME), arbusto, bJ.

98. ${ }^{\circ}$ C. odorata (L.) R.M.King. \& H.Rob., S. Morales S. 595, 791 (FCME), arbusto, bQ, bcQ.

99. Cosmos sulphureus Cav., S. Morales S. 307 (FCME), hierba, btc.

100. CDahlia coccinea Cav., S. Morales S. 331, 1124 b (FCME), hierba, btc, bcQ.

101. ${ }^{\circ}$ D. tenuicaulis P.D.Sorensen, S. Morales S. 168 (FCME), hierba, bQ.

102. + Dyssodia tagetiflora Lag, S. Valencia A. 5268 (FCME), hierba, btc.

103. OFlorestina pedata (Cav.) Cass., S. Morales S. 37 (FCME), hierba, btc.

104. (C)aleana pratensis (Kunth) Rydb., S. Morales S. 332 (FCME), hierba, btc.

105. Galinsoga parviflora Cav., S. Morales S. 273, 351, 471 (FCME), hierba, bJ, bQ, bcQ.

106. (CGuardiola mexicana Bonpl., S. Morales S. 714 (FCME), arbusto, bQ.

107. G. tulocarpus A. Gray., S. Morales S.116, 269 (FCME), hierba, bQ, bJ.

108. Hymenostephium cordatum (Hook. \& Arn.) S.F.Blake, S. Morales S. 600 (FCME), hierba, bJ.

109. Jaegeria hirta (Lag.) Less., S. Morales S. 498 (FCME), hierba, bcQ.

110. (C) Lasianthaea crocea (A.Gray) K.M.Becker, S. Morales S. 638 (FCME), arbusto, bQ.

111. (C) ${ }^{\circ}$ L. helianthoides DC. var. helianthoides, S. Morales S. 69 (FCME), arbusto,bQ.

112. Melampodium microcephalum Less., S. Morales S. 758 (FCME), hierba, bJ.

113. ${ }^{\circ}$ M. dicoelocarpum B.L.Rob., O. Hinojosa E. 350 (FCME), hierba, btc.

114. (OM. divaricatum (Rich.) DC., S. Morales S. 20 (FCME), hierba, btc.

115. OM. gracile Less., S. Valencia A. 5220 (FCME), hierba, btc.

116. $\bullet$ M. linearilobum DC., C. Pringle 10065 (MEXU), hierba, btc.

117. ${ }^{\circ}$ M. longipilum B.L.Rob., S. Morales S. 265 (FCME), hierba, b).

118. M. montanum Benth., S. Morales S. 472, 479 (FCME), hierba, bpe, bQ.

119. Montanoa bipinnatifida (Kunth) Koch, S. Morales S. 567 (FCME), arbusto, bQ.

120. ${ }^{\circ}$ M. frutescens Mairet ex DC., S. Morales S.732 (FCME), árbol, bQ.

121. M. sp., S Morales S.359 (FCME), árbol, bQ.

122. (O)topappus epaleaceus Hemsl., J. L. Panero 2275 (MEXU), hierba, btc.

123. ( $\bullet$ O. imbricatus (Sch.Bip.) S.F.Blake., S. Morales S. 56 (FCME), arbusto, btc.

124. ${ }^{\circ}$ Pectis haenkeana (DC.) Sch.Bip., S. Morales S. 734 (FCME), hierba, bQ.

125. •Perymenium macrocephalum Greenm., J. L. Panero 2274 (MEXU), hierba, btc.

126. Pinaropappus roseus (Less.) Less., S. Morales S. 849 (FCME), hierba, bcQ.

127. Piqueria trinervia Cav., S. Morales S. 263, 474, 725 (FCME), hierba, bJ, bcQ, bQ.

128. (CPittocaulon bombycophole (Bullock) H.Rob. \& Brettell, S. Morales S. 814, 835 (FCME), arbusto, btc, bJ.

129. ${ }^{\circ}$ P. velatum (Greenm.) H.Rob. et Brettell., O. Hinojosa E. 334 (FCME), arbusto, bQ.

130. Psacalium peltatum (Kunth) Cass., S. Morales S. 1136 (FCME), hierba, bcQ.

131. ' Pseudelephantopus spicatus (B.Juss. ex Aubl.) Rohr ex Gleason, S. Valencia A. 5270 (FCME), hierba, btc. 
Anexo 1. Continuación

132. Pseudognaphalium attenuatum (DC.) Anderb., S. Morales S. 700 (FCME), hierba, bcQ.

133. CSanvitalia procumbens Lam., S. Morales S. 296 (FCME), hierba, bJ.

134. OSchkuhria pinnata (Lam.) Kuntze ex Thell var. wislizeni (A.Gray) B.L.urner, S. Morales S. 144 (FCME), hierba, bQ.

135. Sclerocarpus divaricatus (Benth.) Benth. \& Hook. f. ex Hemsl., S. Morales S. 408 (FCME), hierba, btc.

136. • S. papposus (Greenm.) Feddema, J.L. Panero 614 (MEXU), hierba, btc.

137. Simsia sp., S. Morales S. 629 (FCME), hierba, bQ.

138. Sonchus oleraceus L., S. Morales S. s.n. (FCME), hierba, btc.

139. CStevia aschonborniana Sch. Bip., S. Morales S. 694, 765 (FCME) hierba, btc, bJ.

140. S. caracasana DC., S. Morales S. 757 (FCME), arbusto, bQ.

141. S. eupatoria (Spreng.) Willd., S. Morales S. 477 (FCME), hierba, bcQ.

142. S. micrantha Lag., S. Morales S. 469 (FCME), hierba, bcQ.

143. S. origanoides Kunth, S. Morales S. 495, 514 (FCME), hierba, bcQ, bQ.

144. S. ovata Willd., S. Morales S. 570, 671 (FCME), arbusto, bQ, btc.

145. S. serrata Cav., S. Morales S. 577 (FCME), arbusto, bQ.

146. OS. trifida Lag., M Vargas 1 (FCME), S. Morales S. 852 (FCME), hierba, bQ, bcQ.

147. ${ }^{\circ}$ S. triflora DC., S. Morales S. 1196 (FCME), arbusto, btc.

148. ${ }^{\circ}$ S. viscida Kunth, S. Morales S. 513 (FCME), hierba, bQ.

149. CTagetes erecta L., S. Morales S. 490 (FCME), hierba, bcQ.

150. T. filifolia Lag., S. Morales S. 340 (FCME), hierba, bQ.

151. T. lucida Cav., S. Morales S. 100 (FCME), hierba, bQ.

152. T. subulata Cerv., S. Morales S. 381 (FCME), hierba, btc.

153. (OT. tenuifolia Cav., S. Morales S. 199 (FCME), hierba, bQ.

154. Tithonia rotundifolia (Mill.) S.F.Blake, S. Morales S.605 (FCME), hierba, bJ.

155. (C+ T. tubiformis (Jacq) Cass., S. Morales S. 730 (FCME), hierba, bQ.

156. Tridax coronopifolia (Kunth) Hemsl., S. Valencia A. 4879 (FCME), S. Morales S. 81 (FCME), hierba, bQ, btc.

157. T. mexicana A.M.Powell, S. Morales S. 223 (FCME), hierba, btc.

158. OVerbesina crocata (Cav.) Less., S. Valencia A. 4882 (FCME), hierba, btc.

159. ${ }^{\circ}$ V. fastigiata B.L.Rob. \& Greenm., O. Hinojosa E. 335 (FCME), arbusto, btc.

160. ${ }^{\circ}$ V. virgata Cav., S. Morales S. 685 (FCME), arbusto, btc.

161. Vernonia alamanii DC., S. Morales S. 829 (FCME), hierba, bcQ.

162. Viguiera cordata (Hook. \& Arn.) D’Arcy, S. Morales S. 661 (FCME), hierba, bJ, bQ.

163. Zinnia violacea Cav., S. Morales S. s.n. (FCME), hierba, bQ.

\section{Begoniaceae}

164. - Begonia balmisiana Ruiz ex Klotzch, R. M. Fonseca s.n. (FCME), hierba, btc.

165. B. biserrata Lindl., S. Morales S. 982 (FCME), hierba, bJ.

166. B. gracilis Kunth, S. Morales S. 485 (FCME), hierba, bcQ.

167. ${ }^{\circ}$ B. incarnata Link \& Otto, S. Morales S. 293 (FCME), hierba, bJ.

168. (CB. monophylla Pav. ex A. DC., S. Morales S. 47, 957 (FCME), hierba, btc, bJ.

169. ${ }^{\circ}$ B. oaxacana A.DC., S. Morales S. 106, 191, 993 (FCME), hierba, bQ.

\section{Betulaceae}

170. Alnus jorullensis Kunth, S. Morales S. 1140 (FCME), árbol, bcQ.

\section{Bignoniaceae}

171. - Arrabidaea patellifera (Schltdl.) Sandwith, S. Morales S. s.n. (FCME), bejuco, btc

172. - Astianthus viminalis (Kunth) Baill., S. Morales S. 86. (FCME), árbol, btc

173. CCrescentia alata Kunth, S. Valencia A. 4798 (FCME), árbol, btc.

174. • Handroanthus impetiginosus (Mart. ex DC.) Standl., S. Morales S. 842, árbol, btc

175. • Paragonia pyramidata (Rich.) Bureau, S. Morales S. 415 (FCME), arbusto btc

176. (OPithecoctenium crucigerum (L.) A.H.Gentry, S. Valencia A. 4809 (FCME), bejuco, btc

177. ${ }^{\circ}$ Tabebuia rosea (Bertol.) A.DC., V. Acosta A. 1 (FCME), árbol, btc.

178. Tecoma stans (L.) Juss. ex Kunth, S. Morales S. s.n (FCME), arbusto, bJ.

\section{Boraginaceae}

179. ${ }^{\circ}$ Antiphytum caespitosum I.M.Johnst., S. Valencia A. 4854 (FCME), hierba, bQ.

180. CCordia morelosana Standl., M. Gutiérrez N. 2 (FCME), árbol, btc. 
Flora y Vegetación de BuenaVista de CuÉllar

Anexo 1. Continuación

181. Ehretia tinifolia L., M. Gutiérrez 1 (FCME), árbol, btc.

182. ${ }^{\circ}$ Heliotropium fallax I.M.Johnst, S. Morales S. 24 (FCME), hierba, btc.

183. H. limbatum Benth., S. Morales S. 942 (FCME), hierba, bQ.

184. OH. pringlei B.L.Rob., S. Morales S. 251 (FCME), hierba, bQ, bJ.

185. H. procumbens Mill., M. Luján 2 (FCME), hierba, btc.

186. Hydrolea spinosa L., A. Andrade R. 2 (FCME), hierba, bQ.

187. Lennoa madreporoides Lex., S. Morales S. 1103 (FCME), hierba, btc.

188. CTournefortia hirsutissima L., S. Morales S. 978 (FCME), árbol, bJ.

189. ${ }^{\circ}$ T. mutabilis Vent., S. Morales S. 636 (FCME), arbusto, bJ.

190. ${ }^{\circ}$ Varronia curassavica Jacq., S. Valencia A. 4800 (FCME), arbusto, btc.

191. Wigandia urens (Ruiz et Pav.) Kunth, S. Morales S. 763, 833 (FCME), arbusto, bJ, bcQ.

\section{Burseraceae}

192. Bursera aptera Ramirez, S. Morales S. 1066 (FCME), árbol, bQ.

193. B. ariensis (Kunth) McVaugh \& Rzed., S. Morales S. 316 (FCME), árbol, btc.

194. (OB. bicolor (Willd. ex Schltdl.) Engl., S. Valencia A. 4860 (FCME), árbol, btc.

195. (CB. bipinnata (DC.) Engl., S. Valencia A. 4848 (FCME), árbol, bQ.

196. CB. copallifera (DC.) Bullock, S. Valencia A. 3637, 4804 (FCME), árbol, btc.

197. (CB. fagaroides (Kunth) Engl., S. Valencia A. 4847, 4858, (FCME), árbol, bQ, btc.

198. ${ }^{\circ}$ B. glabra (Jacq.) Triana 6 Planch., J.C. Soto 8921 (MEXU), árbol, btc.

199. (CB. glabrifolia (Kunth) Engl., S. Valencia A. 4817, 4840 (FCME), árbol, bQ, bJ, btc.

200. (CB. grandifolia (Schltdl.) Engl., S. Morales S. 1086 (FCME), árbol, btc.

201. (CB. schlechtendalii Engl., S. Valencia A. 4804, 4811 (FCME), árbol, btc.

\section{Cactaceae}

202. +Coryphantha elephantidens (Lem.) Lem., S. Morales S. s.n. (FCME), btc, LC. A.

203. Mammillaria sp., S. Morales S. 800 (FCME), btc.

204. Opuntia tomentosa Salm-Dyck, S. Morales S. 858 (FCME), btc.

205. (OO. velutina F.A.C.Weber, S. Morales S. 888 (FCME), btc.

\section{Cannabaceae}

206. Celtis caudata Planchos, S. Valencia A. 4553 (FCME), M. Gutiérrez N. 3 (FCME), árbol, btc, bJ.

\section{Caprifoliaceae}

207. Valeriana urticifolia Kunth, S. Morales S. 99 (FCME), hierba, bQ.

\section{Caricaceae}

208. C) acaratia mexicana A.DC., S. Morales S. 1073 (FCME), árbol, btc.

209. +\%arilla nana (Benth.) McVaugh, S. Morales S. 999 (FCME), hierba, bQ.

\section{Caryophyllaceae}

210. ${ }^{\circ}$ Drymaria laxiflora Benth., S. Morales S. 176, 1169 (FCME), hierba, bQ, bcQ.

211. (C) D. villosa Schltdl. \& Cham., F. Lorea 3198 (FCME), hierba, bQ.

212. ${ }^{\circ}$ Minuartia moehringiodes (Moc. \& Sessé ex DC.) Mattf., S. Morales S. 523 (FCME), hierba, bQ.

\section{Celastraceae}

213. - Semialarium mexicanum (Miers) Mennega, (FCME), bejuco, btc.

214. Hippocratea celastroides Kunth, A. Hernández O. 1 (FCME), bejuco, btc.

\section{Combretaceae}

215. Combretum fruticosum (Loefl.) Stuntz, S. Morales S. 646 (FCME), árbol, btc.

\section{Convolvulaceae}

216. (C) Calycobolus nutans (M.Martens \& Galeotti) Austin., S. Valencia A.4554 (FCME), hierba, btc.

217. (C) Cuscuta tinctoria Mart. ex Engelm., S. Valencia A. 5226 (FCME), hierba, btc.

218. CEvolvulus alsinoides (L.) L., S. Valencia A., 3388, 4832 (FCME), hierba, bQ.

219. Clpomoea arborescens (Bonpl. ex Willd.) G. Don, S. Morales S. s.n (FCME), árbol, btc. 
Anexo 1. Continuación

220. Cl. bracteata Cav., Y. Dávila 4 (FCME), bejuco, btc.

221. (CI. capillacea (Kunth) G.Don, S. Morales S. 450 (FCME), hierba, btc.

222. (C) cholulensis Kunth, S. Morales S. 290 (FCME), hierba, bJ.

223. I. conzatti Greenm., S. Morales S. 813 (FCME), bejuco, btc.

224. I. costellata Torr., S. Valencia A. 5223 (FCME), hierba,btc.

225. ${ }^{\circ}$ I. dimorpophylla Greenm., S. Morales S. 1164 (FCME), bejuco, bcQ.

226. I. elongata Choisy, S. Valencia A. 3366 (FCME), hierba, bQ.

227. I. murucoides Roem. \& Schult., S. Morales S.752, 737 (FCME), árbol, bJ, bQ.

228. I. nil (L.) Roth, S. Valencia A. 5259 (FCME), hierba, btc.

229. ${ }^{\circ}$ I. oocarpa Benth., S. Morales S. 271 (FCME), hierba, bJ.

230. I. purpurea (L.) Roth., S. Morales S. 452 (FCME), hierba, btc.

231. ${ }^{\circ}$ I. robinsonii House, S. Morales S. 1068 (FCME), bejuco, btc.

232. I. suaveolens (M.Martens \& Galeotti) Hemsl., S. Morales S. 53 (FCME), hierba, btc.

233. I. suffulta (Kunth) G Don, S. Morales S. s.n (FCME), hierba, bQ.

234. (CI. ternifolia Cav., S. Morales S. 1081 (FCME), hierba, btc.

235. OI. tricolor Cav., S. Morales S. 455 (FCME), hierba, btc.

236. ${ }^{\circ}$ I. triloba L., S. Morales S. 311 (FCME), hierba, btc.

237. (O) Operculina pteripes (G.Don) O’ Donell, S. Morales S. 1050 (FCME), bejuco, btc.

\section{Campanulaceae}

238. Diastatea micrantha (Kunth) McVaugh, S. Morales S. 767 (FCME), hierba, bJ.

239. D. tenera (A.Gray) McVaugh, A. Vera O. 2 (FCME), S. Morales S. 474 (FCME), hierba, bQ, bcQ.

240. D. virgata Scheidw., S. Morales S. 657 (FCME), S. Valencia A. 3391 (FCME), hierba, bJ, bQ.

241. Lobelia laxiflora Kunth, M. Lara 2 (FCME), hierba,bQ.

\section{Crassulaceae}

242. (OEcheveria gibbiflora Lindl., S. Morales S. 444 (FCME), hierba, bJ.

243. ${ }^{\circ}$ Sedum jaliscanum S.Watson, S. Morales S. 126, 1131, 1150 (FCME), hierba, bQ, bJ, bpe.

244. ${ }^{\circ}$ S. quevae Hamet, S. Morales S. 460, 712 (FCME), hierba, bJ,bQ.

\section{Cucurbitaceae}

245. Cyclanthera dissecta (Torr. \& A.Gray) Arn., S. Morales S. 1119 (FCME), hierba, btc.

246. Echinopepon pubescens (Benth.) Rose, S. Morales S. 222 (FCME), hierba, btc.

247. ${ }^{\circ}$ Schizocarpum filiforme Schrad., S. Morales S. 735, 1129 (FCME), hierba, bQ, bJ.

\section{Cytinaceae}

248. Bdallophytum americanum (R.Br.) Eichler ex Solms, S. Morales S. 979 (FCME), hierba, bJ.

\section{Ericaceae}

249. Arbutus xalapensis Kunth, S. Morales S.462, 845 (FCME), árbol, bQ, bcQ, LC.

250. Arctostaphylos oppositifolia Parry, S. Morales S. 936 (FCME), árbol, bQ.

\section{Euphorbiaceae}

251. ${ }^{\circ}$ Acalypha phleoides Cav., S. Valencia A. 4803 (FCME), hierba, btc.

252. Acalypha sp., G. Rios 7 (FCME), hierba, bQ.

253. (C) Cnidoscolus angustidens Torr., S. Morales S. s.n. (FCME), hierba, btc.

254. ${ }^{* o}$ C. rostratus Lundell, S. Valencia A. 4793 (FCME), hierba, btc.

255. Croton adspersus Benth., S. Morales S. 923, 1042 (FCME), arbusto, btc, bcQ.

256. (OC. ciliatoglandulifer Ortega. S. Morales S. 44, 458 (FCME), arbusto, btc, bJ.

257. ${ }^{\circ}$ C. mazapensis Lundell, A. Hernández O. 5 (FCME), arbusto, btc.

258. OC. morifolius Willd., S. Valencia A. 4822 (FCME), arbusto, bQ.

259. (C) Dalembertia populifolia Baill, J.C. Soto 8907 (MEXU), bejuco, btc.

260. Euphorbia ariensis Kunth, S. Morales S. 1025 (FCME), hierba, bcQ.

261. ${ }^{\circ}$ E. calyculata Kunth, S. Morales S. 701 (FCME), árbol. bQ.

262. E. hirta L., S. Morales S. 305, 1082 (FCME), hierba, btc, bJ.

263. E. macropus (Klotzsch \& Garcke) Boiss, S. Morales S. 35, 133, 1017 (FCME), hierba, btc, bQ, bcQ.

264. E. prostrata Aiton, S. Morales S. 470 (FCME), hierba, bJ. 
Flora y VEGETACIÓN DE BuENAVista de CuÉllaR

Anexo 1. Continuación

265. (OE. schlechtendalii Boiss, S. Morales S. 837 (FCME), arbusto, bJ.

266. OManihot angustiloba (Torr.) Muell, S. Morales S. 1036 (FCME), hierba. bcQ.

\section{Fabaceae}

267. (CAcacia cochliacantha Humb. \& Bopnl. ex Willd., S. Valencia A. 4810 (FCME), arbusto, btc.

268. (CA. farnesiana (L.) Willd., S. Morales S. 92 (FCME), arbusto, btc.

269. • A. macracantha Humb et Bonpl. ex Willd., C. Catalán s.n. (MEXU), árbol, btc.

270. A. pennatula (Schltdl. \& Cham.) Benth., S. Morales S. 256 ,362, 593 (FCME,), árbol, bJ, btc, bQ.

271. (CAcaciella angustissima (Mill.) Britton et Rose, S. Valencia A. 4826 (FCME), arbusto, bQ.

272. $\bullet$ A. sousae (L.Rico) L.Rico., (FCME) arbusto, btc.

273. (CAeschynomene americana L. var. flabellata Redd., S. Valencia A. 5222 (FCME), hierba, btc.

274. ${ }^{\circ}$ Bauhinia andrieuxii Hemsl., S. Morales S. 946 (FCME), arbusto, bQ.

275. ํㅡㄹ Brongniartia montalvoana Dorado \& Arias, S. Valencia A. 4819 (FCME), arbusto, bQ.

276. Brongniartia podalyrioides Kunth, S. Morales S. s.n (FCME), hierba, bQ.

277. (C) Calliandra houstoniana (Mill.) Standl., S. Morales S. 1024 (FCME), arbusto, bcQ.

278. Cologania broussonetii (Balb.) DC., S. Morales S. s.n. (FCME), hierba, btc.

279. Coursetia caribaea (Jacq.) Lavin, S. Valencia A. 5280 (FCME), hierba, btc.

280. Crotalaria cajanifolia Kunth, S. Morales S.128 (FCME), arbusto, btc.

281. C. pumila Ortega, S. Valencia A. 5225 (FCME), hierba, btc, LC.

282. Dalbergia congestiflora Pittier, S. Valencia A. 5281 (FCME), árbol, btc. P.

283. CDalea cliffortiana Willd., S. Morales S. 2 (FCME), hierba. bQ.

284. D. foliolosa (Aiton) Barneby, S. Morales S.478 (FCME), hierba. bQ.

285. D. humilis G.Don, S. Morales S. 562 (FCME), hierba. bQ.

286. ${ }^{\circ}$ D. sericea Lag., S. Morales S.712 (FCME), hierba. bQ.

287. D. sp., O. Tabares 1 (FCME), hierba, bQ.

288. ${ }^{\circ}$ Desmodium plicatum Schltdl. \& Cham., A. Hernández 2 (FCME), hierba, bQ.

289. D. sp., M. Fonseca 2 (FCME), hierba, bQ.

290. ${ }^{\circ}$ Diphysa ormocarpoides (Rudd.) Sousa \& Antonio, O. Tabares 2 (FCME), arbusto, bQ.

291. D. puberulenta Rydb., S. Morales S. 792 (FCME), arbusto, bQ.

292. D. suberosa S.Watson, S. Valencia A. 4821, 4861 (FCME), arbusto, btc.

293. Erythrina lanata Rose, S. Morales S. 912 (FCME), árbol. bJ.

294. Eysenhardtia platycarpa Pennell \& Saff., S. Morales S. 61, 1018 (FCME), árbol, btc, bJ.

295. ${ }^{\circ}$ Galactia viridiflora (Rose) Standl., S. Morales S. 167 (FCME), bejuco, bQ.

296. Haematoxylum brasiletto H.Karst., S. Morales S. 941 (FCME), árbol, btc.

297. Harpalyce sousai Arroyo, S. Morales S. (FCME), árbol, bQ.

298. Indigofera miniata Ortega, S. Morales S. 68 (FCME), hierba, btc.

299. + Inga vera Willd. var. eriocarpa (Benth.) J.León, S. Morales S. 810 (FCME), árbol, btc.

300. CLeucaena esculenta (DC.) Benth, S. Morales S. 651 (FCME), árbol, btc.

301. • Lonchocarpus caudatus Pitier, F. Terán 492 (MEXU), árbol, LC.

302. Lupinus elegans Kunth, S. Morales S. s.n. (FCME), hierba, btc.

303. (C) Lysiloma divariacatum (Jacq.) J.F.Macbr., A. Carrizosa V. 1 (FCME), árbol, btc.

304. CL. tergeminum Benth., S. Morales S. 1183 (FCME), árbol, btc.

305. CMacroptilium atropurpureum (Moc. \& Sessé ex DC.) Urb., S. Morales S. 598 (FCME), hierba, bJ.

306. M. gibbosifolium (Ortega) A.Delgado, S. Morales S. 392 (FCME), hierba, bQ.

307. Marina sp., S. Morales S. 537 (FCME), hierba, bQ.

308. Mimosa albida Humb. \& Bonpl ex Willd var albida, S. Morales S. 105, 854 (FCME), arbusto, bQ, bcQ, LC.

309. CM. polyantha Benth., D. Rios 2 (FCME), árbol, bQ.

310. $\bullet$ M. polydactyla Bonpl. ex Willd, J.C. Soto 8727 (MEXU), arbusto, btc.

311. CNissolia fruticosa Jacq., S. Valencia A. 4871 (FCME), S. Morales S. 259 (FCME), bejuco, btc, bJ.

312. N. laxior (B.L.Rob.) Rose, S. Morales S. 640 (FCME), bejuco, btc.

313. ${ }^{\circ}$ N. leiogyne Sandwith, S. Morales S. 84 (FCME), bejuco, btc.

314. CPachyrhizus erosus (L.) Urb., S. Morales S. 211 (FCME), hierba, btc.

315. Phaseolus micranthus Hook et Arn., S. Valencia A. 5226 (FCME), hierba, btc.

316. OP. vulgaris L., S. Morales S. 1128 (FCME), hierba, btc.

317. Piscidia grandifolia (Donn. Sm.) I.M.Johnst., S. Morales S. s.n. (FCME), árbol, btc.

318. (Pithecellobium dulce (Roxb.) Benth., S. Valencia A. 4870 (FCME), árbol, btc.

319. Ramirezella lozanii (Rose) Piper, S. Morales S.465 (FCME), hierba, btc. 
Anexo 1. Continuación

320. (C) Senna nicaraguensis (Benth.) H.S.Irwin \& Barneby, S. Morakes S. 58 (FCME), arbusto, btc.

321. (C) S. racemosa var. sororia H.S.Irwin \& Barneby, F. Terán s.n. (MEXU), arbusto, btc.

322. (C) S. skinneri (Benth.) H.S.Irwin \& Barneby, E. Martínez S. s.n. (MEXU), arbusto, btc.

323. (C•S. uniflora (Mill) H.S.Irwin \& Barneby, E. Martínez S. s.n. (MEXU), arbusto, btc.

324. Trifolium amabile Kunth, S. Morales S. 124, 778 (FCME), hierba, bQ, bcQ.

325. ${ }^{\circ}$ V. vexilata (L.) A. Rich., S. Morales S. 94 (FCME), hierba, btc.

326. Zornia reticulata Sm., S. Morales S. 188 (FCME), hierba, bQ.

\section{Fagaceae}

327. Quercus candicans Née, S. Morales S. 1124 (FCME), árbol, bcQ.

328. Q. castanea Née, S. Morales S. 589 (FCME), árbol, bQ.

329. Q. conspersa Benth., S. Valencia A. 3420 (FCME), árbol, bQ.

330. C+ Q. glaucoides M.Martens ex Galeotti, S. Valencia A. 4843 (FCME), S. Morales S. 239 (FCME), árbol, bQ, btc.

331. + Q. magnoliifolia Née, S. Valencia A. 3383, 4831 (FCME), árbol, bQ.

332. + Q. obtusata Bonpl., S. Morales S. 535 (FCME), árbol, bQ.

333. + Q. urbanii Trel., S. Morales S. 590 (FCME), árbol, bQ.

\section{Gentianaceae}

334. Zeltnera quitense (Kunth) G.Mans., S.Morales S. 738 (FCME), hierba, bQ.

\section{Gesneriaceae}

335. Achimenes glabrata (Zucc.) Fritsch, S. Morales S. 383 (FCME), hierba, bQ.

336. (CA. grandiflora (Schltdl.) DC., S. Morales S. 23 (FCME), hierba, btc.

337. * o A. woodii C.V.Morton, S. Morales S. 1168 (FCME), hierba, bpe.

338. ${ }^{\circ}$ Eucodonia verticillata (M.Martens \& Galeotti) Wiehler, S. Morales S. 956 (FCME), hierba, bJ.

\section{Hernandiaceae}

339. (C) Grocarpus jatrophifolius Domin., S. Morales S. 649 (FCME), árbol, btc.

\section{Hypericaceae}

340. Hypericum sp., M. Lara 4 (FCME), hierba, bQ.

\section{Lamiaceae}

341. +Asterohyptis stellulata (Benth.) Epling, S. Morales S. 404, 553 (FCME), arbusto, btc, bQ.

342. Hyptis mutabilis (Rich.) Briq., S. Morales S. 552 (FCME), arbusto, bQ.

343. ${ }^{\circ}$ H. urticoides Kunth, S. Morales S. 270 (FCME), hierba, bJ.

344. Salvia breviflora Moc. \& Sessé, S. Morales S. 261 (FCME), hierba. bJ.

345. S. inconspicua Bertol., S. Valencia A. 5278 (FCME), hierba, btc.

346. ${ }^{\circ}$ S. Iongispicata M.Martens \& Galeotti, S. Morales S. 38 (FCME), hierba, btc.

347. S. mexicana L., S. Morales S. s.n. (FCME), hierba, bQ.

348. (CS. polystachia Cav., S. Morales S. 1182 (FCME), arbusto,bQ.

349. (C). purpurea Cav., S. Morales S. 505, 624 (FCME), hierba, bcQ, bJ.

350. S. sessei Benth., G. Castillo 9 (FCME), arbusto,bQ.

351. ${ }^{\circ}$ S. scaposa Epling, S. Morales S. 625 (FCME), hierba, bJ.

352. ${ }^{\circ}$ S. villosa Fern., J.C. Soto 8915 (MEXU), hierba, btc.

353. Scutellaria caerulea Moc. \& Sessé ex Benth., S. Morales S. 1016 (FCME), hierba. bQ.

354. ${ }^{\circ}$ S. dumetorum Schltdl., S. Morales S. 1151 (FCME), hierba, btc.

355. ${ }^{\circ}$ S. pseudocoerulea Briq., S. Morales S. 584 (FCME), hierba, bQ.

\section{Lentibulariaceae}

356. ${ }^{\circ}$ Pinguicula crenatiloba A.DC., S. Valencia A. 3381 (FCME), hierba, bQ.

357. P. heterophylla Benth., S. Valencia A. 4836 (FCME), hierba, bQ.

358. P. moranensis Kunth, S. Morales S. 1019 (FCME), hierba, bQ.

359. P. parvifolia B.L.Rob., S. Morales S. 932 (FCME), hierba, bQ. 
Flora y VEgetación de BuEnAVista de CuÉllar

Anexo 1. Continuación

\section{Loasaceae}

360. • Eucnide hirta (Pav. ex G.Don) H.J.Thomps. \& W.R.Ernst, M.C. Carlson 3031 (MEXU), hierba, btc.

361. Gronovia scandens L., S. Morales S. 236 (FCME), hierba, btc.

\section{Loganiaceae}

362. Buddleja sessiliflora Kunt, O. Tabares 3 (FCME), arbusto, btc.

\section{Loranthaceae}

363. ${ }^{\circ}$ Cladocolea pedicellata Kuijt, S. Morales S.s.n. (FCME), arbusto, bQ.

364. (OPsittacanthus calyculatus (DC.) D.Gon, S. Morales S, 400 (FCME), arbusto, btc

365. P. palmeri (S. Watson) Barlow \& Wiens, E. Martínez 52 (FCME), arbusto, bcQ.

366. ${ }^{\circ}$ P. schideanus (Schlecht \& Cham.) Blume ex Schult., S. Morales S. 1061 (FCME), arbusto, btc.

367. ' Struthanthus interruptus (Kunth) G.Don, S. Morales S. 995 (FCME), arbusto, bQ.

\section{Lythraceae}

368. Cuphea avigera B.L.Rob. \& Seaton, S. Morales S., 308, 581 (FCME), hierba, btc, bQ.

369. ${ }^{\circ}$ C. calophylla Cham. et Schltdl. (MEXU), hierba, btc

370. C. heterophylla Benth., S. Morales S. 366 (FCME), hierba, bQ.

371. C. hyssopifolia Kunth, S. Morales S. (FCME), hierba, bJ.

372. C. hookeriana Walp., S. Morales S. 192 (FCME), hierba, bQ.

373. ${ }^{\circ}$ C. koehneana Rose, S. Morales S. 268, 1116 (FCME), hierba, bJ, bcQ.

374. ${ }^{\circ}$ C. lanceolata W.T.Aiton, S. Morales S. 975 (FCME), hierba, bJ.

375. C. lobophora Koehne, S. Morales S. 70 (FCME), hierba, btc.

376. C. lutea Rose ex Koehne, S. Valencia A. 5223 (FCME), hierba, btc.

377. C. sp., S. Morales S., s.n. (FCME), hierba, bQ.

378. C. tolucana Peyr., S. Morales S. 98 (FCME), hierba, bQ.

379. C. wrightii A.Gray, S. Valencia A. 3367 (FCME), S. Morales S. 333 (FCME) hierba, bQ, btc.

\section{Malpighiaceae}

380. CBunchosia palmeri S.Watson, S. Morales S. 240, 631 (FCME), árbol, btc, bJ.

381. CByrsonima crassifolia (L.) Kunth, S. Valencia A. 4866 (FCME), S. Morales S., 891 (FCME), árbol, btc, bQ.

382. Callaeum coactum D.M.Johnson, A. Hernández O. 4 (FCME), bejuco, btc.

383. (CGalphimia glauca Cav., S. Morales S. 803 (FCME) arbusto, bJ.

384. Gaudichaudia albida Cham. \& Schltdl., S. Valencia A. 5267 (FCME), S. Morales S. 103 (FCME), bejuco, btc, bQ.

385. ${ }^{\circ}$ G. mucronata (Moc. \& Sessé ex DC.) A.Juss., S. Valencia A. 4850 (FCME), bejuco, bQ.

386. OHeteropterys brachiata (L.) DC. , S. Morales S. 335 (FCME), S. Valencia A. 4820 (FCME), bejuco, btc, bQ.

387. Lasiocarpus salicifolius Liebm., S. Valencia A. 5266 (FCME), árbol, btc.

388. ${ }^{\circ}$ Malpighia galeottiana A.Juss, S. Morales S. 47 (FCME), árbol, btc.

389. CM. mexicana A.Juss., S. Morales S. 968 (FCME), árbol, bJ.

390. Mascagnia polybotrya Nied., S. Morales S. s.n. (FCME), bejuco, bQ.

\section{Malvaceae}

391. (CAnoda cristata (L.) Schltdl., S. Morales S. 242 (FCME), hierba, btc.

392. (C) Ceiba aesculifolia (Kunth) Britten \& Baker f., S. Morales S. 878 (FCME), árbol, btc.

393. C. aff. parvifolia Rose, S. Morales S. 1121 (FCME), árbol, btc.

394. (C) Guazuma ulmifolia Lam. O. Rivera H. 1 (FCME), árbol, bQ.

395. CHeliocarpus pallidus Rose, S. Morales S. 647 (FCME), árbol, btc.

396. H. terebinthinaceus (DC.) Hochr., S. Morales S. 726 (FCME), árbol, bQ.

397. ${ }^{\circ}$ Melochia corymbosa (C.Presl) C.FW.Meissn. ex Steud., S. Morales S. 45 (FCME), arbusto, btc.

398. CM. pyramidata (L.) Britt., M. Lujan H. 2 (FCME), arbusto, bQ.

399. (OPseudobombax ellipticum (Kunth) Dugand, A. Carrizosa V. 2 (FCME), árbol, btc.

400. ${ }^{\circ}$ Sida abutifolia Mill., S. Morales S. 234 (FCME), hierba, btc.

401. S. glabra Mill., S. Morales S. 705 (FCME), hierba, bQ.

402. CS. rhombifolia L., S. Morales S. 170 (FCME) hierba,bQ.

403. CTriumfetta semitriloba Jacq., S. Morales S. 233, 275 (FCME), árbol, btc, bJ.

404. CWaltheria americana L., S. Morales S. 334 (FCME), arbusto, btc.

405. W. indica L., S. Valencia A. 4816 (FCME), S. Morales 618, 857 (FCME), sufrutice, bQ, bJ, bcQ. 
SAdDAN Morales-SALDAÑA ET AL.

Anexo 1. Continuación

Martyniaceae

406. CMartynia annua L., S. Morales S. 987 (FCME), hierba, btc.

Melastomataceae

407. Tibouchina sp., S. Morales S. 851 (FCME), hierba, bcQ.

\section{Meliaceae}

408. • Cedrela salvadorensis Standl., C. Catalán 591 (MEXU), árbol, btc.

409. - Melia azedarach L., N. Diego y M. Castro 9288 (FCME, MEXU), árbol, btc.

410. CSwietenia humilis Zucc., S. Morales S. 1050 (FCME), árbol, btc. VU.

411. Trichilia americana (Sessé \& Moc.) T.D. Penn., S. Morales S. 319 (FCME), árbol, btc.

412. CT. hirta L., S. Morales S. 60 (FCME), árbol, btc.

\section{Menispermaceae}

413. Cissampelos pareira L., S. Valencia A. 4863 (FCME), S. Morales S. 1038 (FCME), hierba, btc, bcQ.

\section{Moraceae}

414. (Dorstenia drakena L., S. Morales S. 1104 (FCME), hierba, btc.

415. Ficus cotinifolia Kunth, S. Morales S. 944, 1094, 1196 (FCME), árbol, bQ, btc , bcQ.

416. F. insipida Willd., S. Morales S. 875 (FCME), árbol, btc.

417. (C+ F. petiolaris Kunth, S. Morales S. 836 (FCME), árbol, bJ.

\section{Muntingiaceae}

418. Muntingia calabura L., C. Catalán 738 (MEXU), arbusto, btc.

\section{Nyctaginaceae}

419. (C) Boerhavia coccinea Mill., C. Catalán 695 (FCME), hierba, btc.

420. •B. sp., C. Catalán. s.n. (FCME), hierba, btc.

421. (C) B. erecta L., C. Catalán 694 (FCME), hierba, btc.

422. ${ }^{\circ}$ Mirabilis sanguinea Heimerl. var. sanguinea, S. Morales S. 920 (FCME), hierba. bQ.

\section{Onagraceae}

423. Fuchsia microphylla Kunth, S. Morales S. 580, 1159 (FCME), arbusto, bQ, bcQ.

424. F. parviflora Lindl., J. Gotes 3 (FCME), sufrutice, bQ.

425. Hauya sp., S. Morales S. 1188 (FCME), árbol, btc.

426. Lopezia miniata Lag. ex DC., S. Morales S. 691 (FCME), hierba, btc.

427. L. racemosa Cav., S. Morales S. 298,504, 546 (FCME), hierba, bJ, bcQ, bQ.

428. L. sp., S. Morales S. 1142 (FCME), hierba, bcQ.

429. Ludwigia octovalvis (Jacq.) P. H. Raven, J. Gotes 1 (FCME), arbusto, btc.

430. L. sp., S. Morales S. 86 (FCME), hierba, btc.

\section{Opiliaceae}

431. (CAgonandra racemosa (DC) Standl., S. Valencia A. 4552 (FCME), árbol, btc.

\section{Orobanchaceae}

432. Castilleja gracilis Benth., S. Morales S. 782 (FCME), hierba, bQ.

433. C. tenuiflora Benth., S. Morales S. 907 (FCME), hierba, bJ.

434. CLamourouxia viscosa Kunth, S. Morales S. 910 (FCME), hierba, bJ.

\section{Oxalidaceae}

435. ${ }^{\circ}$ Oxalis angustifolia Kunth, S. Valencia A. 4838 (FCME), hierba, bQ.

436. ${ }^{\circ}$ O. dimidiata Donn. Sm., S. Morales S. 892 (FCME), hierba, bJ.

437. ${ }^{\circ}$ O. jacquiniana Kunth, S. Valencia A. 4801 (FCME), hierba, btc.

438. O. latifolia Kunth, S. Morales S. 899 (FCME), hierba, bJ.

439. ${ }^{\circ}$ O. Iunulata Zucc., S. Valencia A. 4818 (FCME), hierba, bQ.

440. O. tetraphylla Cav., S. Morales S. 898 (FCME), S. Valencia A. 4856 (FCME), hierba bJ, bQ. 
Flora y VEGETACIÓN DE BuEnAVista de CuÉllaR

Anexo 1. Continuación

\section{Passifloraceae}

441. ${ }^{\circ}$ Passiflora colimensis Mast. \& Rose, S. Morales S. s.n (FCME), hierba, bQ.

442. ${ }^{\circ}$ P. sicyoides Schltdl. \& Cham, S. Morales S 285, 341 (FCME), hierba, bJ, btc.

443. Turnera ulmifolia L., S. Morales S. 194, 1128 (FCME), hierba, bQ, bcQ.

\section{Phytolaccaceae}

444. Phytolacca icosandra L. M. Fonseca 4 (FCME), hierba. bQ.

\section{Piperaceae}

445. (C) Peperomia bracteata A.W.Hill, S. Morales S. 57 (FCME), S. Valencia A. 3403 (FCME), hierba, btc, bQ . 446. $+{ }^{\circ}$ P. cavispicata G.Mathieu, S. Morales S. 1123 (FCME), hierba, bJ.

\section{Plantaginaceae}

447. ${ }^{\circ}$ Maurandya erubescens (D.Don) A.Gray, S. Morales S. 972 (FCME), hierba, bJ.

448. Mecardonia procumbens (Mill.) Small., S. Valencia A. 4833 (FCME), hierba, bQ.

449. ${ }^{\circ}$ Russelia polyedra Zucc., S. Valencia A. 4827 (FCME), arbusto, bQ.

450. ${ }^{\circ}$ Russelia pringlei B.L.Rob., S. Morales S. 587 (FCME), hierba, bQ.

451. Russelia retrorsa Greene, S. Morales S. 377 (FCME), hierba, bQ.

\section{Plumbaginaceae}

452. Plumbago scandens L., S. Morales S. 312 (FCME), hierba, btc.

\section{Polemoniaceae}

453. Loeselia glandulosa (Cav.) G.Don., S. Morales S. 655, 750, 773 (FCME), hierba, btc, bJ, bQ.

454. ${ }^{\circ}$ L. caerulea (Cav.) G.Don., S. Morales S. 517 (FCME), hierba, bQ.

455. (CL. mexicana (Lam.) Brand, G. Castillo 6 (FCME), S. Morales S. 816 (FCME), arbusto, bQ btc.

\section{Polygalaceae}

456. ${ }^{\circ}$ Polygala berlandieri S.Watson, S. Valencia A. 3387 (FCME), hierba, bQ.

457. P. compacta Rose, S. Morales S. 27 (FCME), hierba, btc.

458. ${ }^{\circ}$ P. consobrina S.F.Blake, S. Morales S. 951 (FCME), hierba, bJ.

459. P. costaricensis Chodat, S. Morales S. s.n (FCME), hierba, bQ.

460. P. glochidata Kunth, S. Morales S. 1033 (FCME), hierba, bcQ.

461. ${ }^{\circ}$ P. paniculata L., S. Morales S. 963 (FCME), hierba, bJ.

\section{Ranunculaceae}

462. (C)lematis dioica L., S. Morales S. 643 (FCME), bejuco, btc.

463. Ranunculus sierrae-orientalis (L.D.Benson) G.L.Nesom, S. Morales S. 132, 1010 (FCME), hierba, bQ, bcQ.

464. Thalictrum guatemalense C.DC. \& Rose, S. Morales S. 964 (FCME), hierba, bJ.

465. ${ }^{\circ}$ T. pubigerum Benth., S. Morales S. 900 (FCME), hierba, bJ.

\section{Rhamnaceae}

466. (C) Colubrina macrocarpa (Cav.) G.Don, S. Morales S. s.n. (FCME), árbol, bQ.

467. (C) triflora Brongn. ex Sweet, S. Valencia A. 4794 (FCME), árbol, btc.

468. Karwinskia humboldtiana (Schult.) Zucc., S. Morales S. 1176 (FCME), S. Valencia A. 4867 (FCME), árbol, bcQ, btc.

469. ${ }^{\circ}$ K. mollis Schltdl., S. Morales S. s.n. (FCME), árbol, bcQ

470. (우 K. umbellata (Cav.) Schltdl., M. Gutiérrez N. 2. (FCME), árbol, btc.

\section{Rubiaceae}

471. Bouvardia chrysantha Mart., S. Valencia A. 3378, 4830 (FCME), hierba, bQ, btc.

472. ${ }^{\circ}$ B. loeseneriana Standl., S. Valencia A. 4805 (FCME), arbusto, btc. Pr.

473. B. multiflora (Cav.) Schult. \& Schult. f., S. Valencia A. 3428 (FCME), sufrutice, bQ.

474. Chiococca filipes Lundell, S. Valencia A. 3417 (FCME), arbusto, bQ.

475. (C) Crusea calocephala DC., S. Valencia A. 3384 (FCME), hierba, bQ. bJ.

476. ${ }^{\circ}$ C. hispida (Mill.) B.L.Rob., S. Morales S. 167 (FCME), hierba, bQ. Pr.

477. (C). longiflora (Willd. ex Roem \& Schult.) W. R. Anderson, S. Valencia A. 3392 (FCME), hierba, bQ

478. ${ }^{\circ}$ C. megalocarpa (A.Gray) S.Watson, S. Morales S. 213 (FCME), hierba, btc. 
Anexo 1. Continuación

479. Galium mexicanum subsp. mexicanum Kuth, S. Valencia A. 5244 (FCME), hierba, btc.

480. Guettarda sp., S. Valencia A. 4875 (FCME), árbol, btc.

481. Hamelia versicolor A.Gray, R. Cruz Duran 990 (FCME), arbusto, btc.

482. • Paederia ciliata (DC.) Standl., E. Martínez S. 1185 (MEXU), bejuco.

483. Randia capitata DC., S. Morales S. 727 (FCME), árbol, bQ.

\section{Rutaceae}

484. Ptelea trifoliata L., S. Morales S. 1177 (FCME), árbol, btc.

485. Zanthoxylum fagara (L.) Sarg., S. Morales S. $319 b$ (FCME), árbol, btc.

\section{Salicaceae}

486. ${ }^{\circ}$ Neopringlea viscosa (Liebm.) Rose, S. Morales S. 1063 (FCME), árbol, btc.

487. (OProckia crucis P. Browne ex L., S. Morales S. 917 (FCME), árbol, btc.

488. Salix sp., V. Acosta A. s.n. (FCME), árbol, btc.

489. Xylosma flexuosa (Kunth) Hemsl., S. Morales S. (FCME), árbol, bQ.

490. ${ }^{\circ}$ X. intermedium (Seem.) Triana \& Planch., S. Morales S. 1022 (FCME), árbol, bcQ

\section{Santalaceae}

491. ${ }^{\circ}$ Phoradendron pedicellatum (Tiegh.) Kuijt, S. Morales S. 409 (FCME), arbusto, btc.

492. ${ }^{\circ}$ P. reichenbachianum (Seem.) Oliv., E. Martínez A. 53 (FCME), arbusto, bQ.

\section{Sapindaceae}

493. Cardiospermum halicacabum L., S. Morales S. 171 (FCME), hierba, bQ.

494. Dodonaea viscosa (L.) Jacq., S. Morales S. 246, 548 (FCME), arbusto, bJ, bQ.

495. ' Serjania racemosa Schumach., S. Morales S. 834 (FCME), bejuco, bJ.

496. ${ }^{\circ}$ S. triquetra Radlk., S. Morales S. 288 (FCME), bejuco, bJ.

497. ${ }^{\circ}$ Thouinia acuminata S.Watson, S. Morales S. s.n. (FCME), arbusto, bJ.

\section{Sapotaceae}

498. CSideroxylon capiri (A.DC.) Pittier, D. Rios L. 3 (FCME), árbol, btc. A.

\section{Simarubaceae}

499. (CAlvaradoa amorphoides Liebm., A. Vera O. s.n. (FCME), árbol, btc.

\section{Solanaceae}

500. (C) Cestrum nocturnum L., J.C. Soto 8736 (MEXU), hierba, btc.

501. CDatura stramonium L., S. Valencia A. 3410 (FCME), hierba, bQ.

502. ()Jaltomata procumbens (Cav.) J.L.Gentry, S. Morales S. 262 (FCME), hierba, bJ.

503. Nicotiana glauca Graham, S. Morales S. s.n (FCME), bJ.

504. P. nicandroides Schltdl., S. Valencia A. 3408 (FCME), hierba, bQ.

505. ${ }^{\circ}$ P. peruviana L., S. Morales S. 1118 (FCME), hierba, bQ.

506. P. philadelpica Lam., S. Morales S. 488 (FCME), hierba. bcQ.

507. ${ }^{\circ}$ P. pruinosa L., S. Morales S. 286 (FCME), hierba, bJ.

508. Solanum americanum Mill., S. Casasa V. s.n. (FCME), arbusto, bQ.

509. S. angustifolium Mill., S. Morales S. s.n. (FCME), arbusto, btc.

510. ${ }^{\circ}$ S. candidum Lindl., S. Morales S.592 (FCME), arbusto, bQ

511. S. erianthum D.Don, S. Morales S. 75, 295, 1170 (FCME), arbusto, btc, bJ, bpe.

512. S. laurifolium Mill., S. Valencia A. 3382 (FCME), S. Morales S. 918 (FCME), arbusto bQ, btc.

513. S. torvum Sw., S. Morales S. 827 (FCME), arbusto, bcQ.

514. S. mitlense Dunal, S. Morales S. 59 (FCME), árbol, bJ.

515. CS. rostratum Dunal, S. Morales S. 77 (FCME), arbusto, btc.

516. S. umbellatum Mill., S. Morales S. 36,883 (FCME), arbusto, btc, bQ.

\section{Urticaceae}

517. ' Pilea microphylla (L.) Liebm., S. Valencia A. 3406 (FCME), hierba, bQ. 
FLoRA y VEGETACIÓN DE BuENAVISTA DE CuÉllaR

Anexo 1. Continuación

Verbenaceae

518. CLantana achyranthifolia Desf., S. Morales S. 983 (FCME), arbusto, bJ.

519. L. camara L., S. Valencia s.n. (FCME), arbusto, bQ.

520. ${ }^{\circ}$ L. glandulosissima Hayek, S. Morales S. 411 (FCME), arbusto, btc.

521. L. urticifolia Mill., S. Morales S. 10 (FCME), arbusto, btc.

522. Lippia alba (Mill.) N.E.Br. ex Britton \& P.Wilson, S. Morales S. 398, 961 (FCME), arbusto, btc, b).

523. (CL. graveolens Kunth, S. Morales S. 615, 677 (FCME), arbusto, bJ, btc.

524. CVitex mollis Kunth, S. Morales S. 1087 (FCME), árbol, btc.

\section{Violaceae}

525. Hybanthus attenuatus (Bonpl. ex Schult.) Schulze-Menz, S. Morales S. 55,179 (FCME), hierba, btc, bQ.

526. Viola hookeriana Kunth, S. Morales S. 949 (FCME), hierba, bQ.

\section{Vitaceae}

527. ${ }^{\circ}$ Ampelopsis denudata Planch, S. Morales S. (FCME), hierba, bJ.

528. ${ }^{\circ}$ Cissus cacuminis Standl, S. Morales S. s.n. (FCME), bejuco, bQ.

529. ${ }^{\circ}$ C. rhombifolia Vahl, S. Morales S. 541 (FCME), bejuco, btc, bQ.

530. (C) ${ }^{\circ}$ C. verticillata (L.) Nicolson \& C.E.Jarvis, S. Morales S. 945 (FCME), bejuco, bQ. btc.

531. Vitis tiliifolia Bonpl. ex Roem. \& Schult., S. Morales S. 950 (FCME), bejuco, bQ.

\section{Monocotiledóneas}

Asparagaceae

532. • Agave angustiarum Trel., A. García-Mendoza, A. Gutíerrez, S. Franco y A. Castañeda s.n. (MEXU), hierba, btc.

533. A. sp., S. Morales S. s.n (FCME), hierba, b).

534. Echeandia echeandioides (Schltdl.) Cruden, S. Morales S. s.n (FCME), hierba, bQ.

535. ${ }^{\circ}$ E. flexuosa (Cav.) Rose, S. Morales S. 943 (FCME), hierba, bQ.

536. ${ }^{\circ}$ E. reflexa Greenm., S. Morales S. 1118 (FCME), hierba, btc.

537. ${ }^{\circ}$ Manfreda scabra (Ortega) McVaugh, S. Morales S. 1008 (FCME), hierba, bQ.

538. $\bullet$ M. rubescens (Regel \& Ortgies), A. García-Mendoza y A. Gutíerrez s.n. (MEXU), hierba, btc.

539. Polianthes geminiflora (Lex.) Rose, S. Morales S. 1133 (FCME), hierba, bcQ.

\section{Amaryllidaceae}

540. Allium glandulosum Link et Otto, S. Morales S. 1166 (FCME), hierba, bcQ.

541. Hymenocallis sp., S. Morales S. 948 (FCME), hierba, bQ.

542. CMilla biflora Cav., S. Morales S. 748 (FCME), hierba. bQ.

\section{Araceae}

543. Arisaema dracontium (L.) Schott., S. Morales S. 928, 981 (FCME), hierba, bQ, bJ.

\section{Arecaceae}

544. Brahea dulcis (Kunth) Mart., S. Morales S. 297 (FCME), hierba, bJ.

\section{Bromeliaceae}

545. • *Hechtia carlsoniae Burt-Utley \& Utley, A. López y A. Espejo 2844 (UAMIZ), hierba. bJ.

546. Pitcairnia aff. sordida L.B.Sm., S. Morales S. 24, 148, 889 (FCME), hierba, btc, bJ, bQ.

547. (P. karwinskyana Schult. \& Schult. f., S. Morales S. 870 (FCME), hierba, bJ.

548. ${ }^{\circ}$ P. roseana L.B.Sm, S. Valencia A. 4842 (FCME), hierba, bQ.

549. (OTillandsia achyrostachys E.Morren ex Baker, S. Morales S. 771, 806 (FCME), hierba, bQ, b).

550. CT. caput-medusae E.Morren, S. Morales S. 880 (FCME), hierba, bQ.

551. T. fasciculata Sw., S. Morales S. 806 (FCME), hierba, bJ.

552. T. hintoniana L.B.Sm., S. Morales S. (FCME), hierba, bQ.

553. OT. ionantha Planch., S. Morales S. 872 (FCME), S. Valencia A. s.n. (FCME), hierba, bJ, btc.

554. CT. recurvata (L.) L., S. Valencia A. 4873 (FCME), hierba, btc. LC.

555. T. schideana Steud., S. Morales S. 841 (FCME), hierba, bJ.

556. T. sp., S. Morales S. s.n. (FCME), hierba, btc. 
Anexo 1. Continuación

\section{Commelinaceae}

557. (C) Commelina coelestis Willd., S. Morales S. 212 (FCME), hierba. btc.

558. C. diffusa Burm. f., J. Gotes 6 (FCME), hierba, bQ.

559. C. erecta L., S. Morales S. s.n. (FCME), hierba, btc.

560. C. leiocarpa Benth., S. Morales S. s.n. (FCME), hierba, bQ.

561. C. tuberosa L., S. Morales S. 473 (FCME), hierba, bpe, bQ.

562. Gibasis linearis Benth., S. Morales S. s.n. (FCME), hierba, bcQ.

563. + Thyrsanthemum floribundum M.Martens \& Galeotti, S. Morales S. 356 (FCME), hierba, btc, bQ.

564. Tradescantia crassifolia Cav., S. Morales S. s.n. (FCME), hierba, bQ.

565. *Tripogandra amplexans Handlos, S. Morales S. s.n. (FCME), hierba, btc.

566. ' T. amplexicaulis (Klotzsch ex C.B.Clarke) Woodson, S. Morales S. 306, 344 (FCME), hierba, btc, bQ.

567. T. angustifolia (B.L.Rob.) Woodson, S. Morales S. 225, 1184 (FCME), hierba, btc, bcQ.

568. ${ }^{\circ}$ T. purpurascens (S.Schauer) Handlos, S. Morales S. 470 (FCME), hierba, bQ.

\section{Cyperaceae}

569. - Bulbostylis funckii (Steud.) C.B. Clarke, R.M. Fonseca 84 (FCME), hierba, bQ.

570. • B. juncoides (Vahl) Kük. ex Osten, R.M. Fonseca 837 (FCME), hierba, bQ.

571. Cyperus esculentus L., A. Catalán 716 (FCME), hierba, btc.

572. • C. flavescens L., R. M. Fonseca 838 (FCME), hierba, bQ.

573. (C) C. hermaphroditus (Jacq.) Standl., R. M. Fonseca 824 (FCME), hierba, bQ.

574. - C. ischnos Schltdl., R. M. Fonseca 834 (FCME), hierba, bQ.

575. - C. manimae Kunth, R. M. Fonseca 839 (FCME), hierba, bQ.

576. • C. mutisii (Kunth) Andersson, R. M. Fonseca 824 (FCME), hierba, bQ.

577. • C. niger Ruiz \& Pav., R. M. Fonseca 824 (FCME), hierba, bQ.

578. C. seslerioides Kunth, S. Valencia A. 4844 (FCME), hierba, bQ.

579. Eleocharis montana (Kunth) Roemer \& Schultes, G. Roldán D. s.n. (FCME), hierba, bQ.

580. Kyllinga odorata Vahl, S. Morales S. 170 (FCME), hierba, bQ.

581. • Lipocarpha micrantha (Vahl) G. C.Tucker, S. Torres 1591 (FCME), hierba, bQ.

582. • Rynchospora contracta (Nees) J.Raynal, R.M. Fonseca 825 (MEXU), hierba, bQ.

\section{Dioscoreaceae}

583. CDioscorea convolvulacea Schltdl. \& Cham., S. Morales S. 369 (FCME), hierba, btc.

584. CD. galeottiana Martens, S. Morales S. 1052 (FCME), hierba, btc.

585. CD. morelosana Matuda, S. Morales S. 1067 (FCME), hierba, btc.

586. CD. remotiflora Kunth, S. Valencia A. 5262 (FCME), hierba, btc.

\section{Eriocaulaceae}

587. ${ }^{\circ}$ Eriocaulon benthamii Kunth, S. Morales S. 1122 (FCME), hierba, bcQ.

\section{Hypoxidaceae}

588. Hypoxis mexicana Schult. \& Schult. f., S. Morales S. 895 (FCME), hierba, bJ.

\section{Iridaceae}

589. ${ }^{\circ}$ Cardiostigma longispicata (Herb.) Baker, S. Morales S. 1004 (FCME), hierba, bQ.

590. ${ }^{\circ}$ Sisyrinchium tenuifolium Bonpl. ex Willd., S. Valencia A. 4834 (FCME), hierba, bQ.

591. Tigridia meleagris (Lindl.) G.Nicholson, S. Morales S. 1000 (FCME), hierba, bQ.

592. T. sp., S. Morales S. 1193 (FCME), hierba, btc.

\section{Liliaceae}

593. + Bessera elegans Schult. f., S. Valencia A. 3394 (FCME), S. Morales S. 1189 (FCME), hierba, bQ, btc.

594. Calochortus barbatus (Kunth) J.H.Painter, S. Morales S. 64 (FCME), hierba, btc.

595. C. purpureus (Kunth) Baker, S. Morales S. 110 (FCME), hierba, bQ.

\section{Marantaceae}

596. ${ }^{\circ}$ Maranta gibba Sm., S. Morales S. 988 (FCME), hierba, btc. 
Flora y VEGETACIÓN DE BuENAVista de CuÉllaR

Anexo 1. Continuación

\section{Orchidaceae}

597. Bletia gracilis Lodd., S. Morales S. s.n. (FCME), hierba, bQ.

598. B. lilacina A.Rich. \& Galeotti, S. Morales S. 824 (FCME), hierba, bQ.

599. ${ }^{\circ}$ B. macristhmochila Greenm, S. Morales S. 925 (FCME), hierba, bQ.

600. Dichromanthus aurantiacus (La Llave \& Lex.) Salazar \& Soto Arenas, S. Morales S. 78, 1031 (FCME), hierba, btc, bcQ.

601. ${ }^{\circ}$ Habenaria crassicornis Lindl., S. Morales S. 1127 (FCME), hierba, bcQ.

602. H. quinqueseta (Michx.) Eaton, S. Valencia A. 5243 (FCME), hierba, bQ.

603. ${ }^{\circ}$ Liparis vexillifera (La Llave \& Lex.) Cogn., S. Morales S. 1146 (FCME), hierba, bcQ.

604. ${ }^{\circ}$ Malaxis aurea Ames, S. Morales S. 243 (FCME), S. Valencia A. 5234 (FCME), hierba, bJ, btc.

605. ${ }^{\circ}$ M. fastigiata (Rchb. f.) Kuntze, S. Morales S. 1163 (FCME), hierba, bcQ.

606. Mesadenus tenuissimus (L.O.Williams) Garay, J. P. Pánico s.n. (FCME), hierba, btc.

607. Tamayorkis ehrenbergii (Rchb. f.) R. González \& Szlach., S. Morales S. 953, 1126 (FCME), hierba, bJ, bcQ.

\section{Poaceae}

608. Digitaria sp., M. Gutiérrez N. 3 (FCME), hierba, bQ.

609. Ixophorus sp., S. Morales S. s.n. (FCME), hierba, bQ.

610. Otatea acuminata (Munro) C.E.Calderon \& Soderstr., S. Morales S. 1054 (FCME), hierba, bJ.

611. Paspalum notatum A.H.Liogier ex Flüggé, S. Morales S. 154, 238 (FCME), hierba, bQ, btc. 